Acta Zoológica Mexicana (nueva serie), Volumen 37, 1-28.

https://doi.org/10.21829/azm.2021.3712354

Artículo original

\title{
Aspectos ambientales y culturales de los nodos panbiogeográficos prioritarios para mamíferos terrestres del centro-sur de México
}

\section{Environmental and cultural issues of Priority Panbiogeographic Nodes of the terrestrial mammals in the center-southern Mexico}

\section{1,2WILLIE CORONA-MENDOZA, (D) 1*TANIA ESCALANTE}

${ }^{1}$ Grupo de Biogeografía de la Conservación, Departamento de Biología Evolutiva, Facultad de Ciencias, Universidad Nacional Autónoma de México. Circuito Exterior s/n, Coyoacán 04510, México.

${ }^{2}$ Colegio de Geografía, Facultad de Filosofía y Letras, Universidad Nacional Autónoma de México, Circuito Interior s/n, Coyoacán 04510, México.

Editor responsable: José Ramírez-Pulido

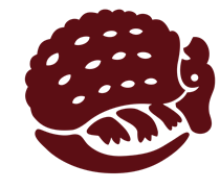

OPEN ACCESS

*Autor corresponsal:

iD Tania Escalante tescalante@ciencias.unam.mx

Cita:

Corona-Mendoza, W., Escalante, T.

(2021) Aspectos ambientales y culturales de los nodos panbiogeográficos prioritarios para mamíferos terrestres del centro-sur de México. Acta Zoológica

Mexicana (nueva serie), 37, 1-28. 10.21829/azm.2021.3712354 elocation-id: e3712354

Recibido: 07 octubre 2020 Aceptado: 03 agosto 2021

Publicado: 13 agosto 2021
RESUMEN. Los Nodos Panbiogeográficos (NP) constituyen uno de los principales aportes de la biogeografía evolutiva a la conservación de la biodiversidad, los cuales se interpretan como la superposición en espacio-tiempo de diferentes fragmentos geobióticos ancestrales, permitiendo identificar prioridades que preservan la riqueza de especies y de orígenes evolutivos. Por otro lado, los mamíferos son un grupo con una importante connotación cultural en las regiones indígenas de México, donde son utilizados principalmente como alimento y medicina. Dado que México es uno de los países con una correlación estrecha entre su gran diversidad biológica y cultural, el objetivo de esta investigación se centró en la priorización y caracterización ambiental y cultural de NP para la conservación de mamíferos en el centro-sur de México. La priorización de la riqueza de especies de los NP se realizó empleando tres criterios, y los NP Prioritarios (NPP) fueron caracterizados ambiental y culturalmente. A partir de siete grupos de NP integrados por 31 NP y 3 NP que no pertenecieron a ningún grupo, se priorizaron 23 NP. La mayoría de los NPP se localizó sobre la provincia biogeográfica de las Tierras Altas de Chiapas, seguida por la Sierra Madre del Sur, mientras que la Faja 
Volcánica Transmexicana tuvo el menor número de NPP. En las dos primeras provincias hubo superposición y aglomeración de NPP, específicamente sobre los Altos de Chiapas y la Sierra Juárez. Estos dos sitios parecen ser las áreas geográficas más complejas desde el punto de vista biogeográfico-evolutivo, físico-geográfico y cultural. En términos de conservación, al conservar los NPP, también se protegerá gran cantidad de recursos naturales y culturales.

Palabras clave: Análisis de Trazos; complementariedad; conservación; Mammalia; pueblos indígenas

ABSTRACT. The Panbiogeographical Nodes (PN) are relevant contributions of the evolutionary biogeography to the biodiversity conservation, which are interpreted as the spatial-temporal overlapping of different ancestral geobiotic fragments, allowing identify priorities to preserve both species and evolutionary origins richness. On the other hand, mammals are a group with an important cultural connotation in the indigenous regions of Mexico, where they are used mainly as food and medicine. Because Mexico is one of the countries with a close correlation between its great biological and cultural diversities, the aim of this research focused on the prioritization and environmental and cultural characterization of PN for the conservation of mammals in centralsouthern Mexico. The prioritization of species richness was carried out employing three criteria, and the Prioritized PN (PPN) were environmental and culturally characterized. From the seven groups of PN integrated by $31 \mathrm{PN}$ and $3 \mathrm{PN}$ without group, we prioritized $23 \mathrm{NP}$. The most of the PPN were located in the Chiapas Highlands biogeographic province, followed by the Sierra Madre del Sur, while the Transmexican Volcanic belt had the lower number of PPN. In the first provinces, there were overlapping and agglomeration of PPN, mainly in the Altos de Chiapas and the Sierra Juárez. These last sites seem to be the geographical areas more complex from biogeographicevolutionary, physical-geographic, and cultural viewpoints. For conservation, the preservation of these PPN will allow to protect many natural and cultural resources.

Key words: Track Analysis; complementarity; conservation; Mammalia; native people

\section{INTRODUCCIÓN}

La biogeografía de la conservación es una subdisciplina de la biogeografía y de la biología de la conservación que se enfoca en la solución de problemas relacionados con la conservación de la biodiversidad, tomando como base la distribución geográfica de los seres vivos (MacDonald, 2003; Whittaker et al., 2005; Escalante \& Morales, 2015). Existen diversos criterios desde el punto de vista biogeográfico para seleccionar áreas de conservación, algunos de los cuales toman en cuenta aspectos evolutivos que involucran factores de espacio y tiempo (Sánchez, 2011; Morrone \& Escalante, 2016). Uno de los enfoques biogeográficos evolutivos es la Panbiogeografía (Croizat, 1958), la cual enfatiza la dimensión espacial de la biodiversidad, permitiendo un mejor entendimiento de los patrones y procesos evolutivos (Craw et al., 1999). Este enfoque asume que la Tierra y la vida evolucionan juntas, debido a los cambios geográficos ocurridos en el pasado (Hull, 1988; Craw et al., 1999). 
La panbiogeografía se implementa a través del Análisis de Trazos (AT; Morrone, 2015), el cual comprende tres pasos: (1) construir trazos individuales para dos o más taxones, a partir de árboles de distancias mínimas que unen a las localidades de registro de un taxón; (2) obtener trazos generalizados donde coinciden dos o más trazos individuales; y (3) identificar Nodos Panbiogeográficos (NP) en las áreas de intersección de dos o más trazos generalizados. En particular, los NP se interpretan como la superposición en espacio-tiempo de diferentes fragmentos geobióticos ancestrales (Morrone, 2001). Para representar los NP se dibuja una " $x$ " encerrada en un círculo, indicando así un punto de convergencia y superposición, lo cual es acorde con los principios de diseño gráfico, sin embargo, carecen de dimensión espacial (Fortino \& Morrone, 1997; Miguel-Talonia \& Escalante, 2013). Existe la posibilidad de encontrar áreas geográficas con alta concentración de NP, que han sido nombradas como "super-nodos" o "áreas nodo-diversas", las cuales son extremadamente complejas desde los puntos de vista geológico y biótico (Miguel-Talonia \& Escalante, 2013).

El AT ha sido aplicado a la conservación biótica (Morrone, 1999; Contreras et al., 2001; García-Barros et al., 2002), ya que al estudiar la biodiversidad desde una perspectiva evolutiva, se identifican prioridades en las áreas a ser conservadas, mismas que permiten preservar tanto la riqueza de especies como la riqueza de orígenes evolutivos (Morrone \& Espinosa-Organista, 1998; Morrone, 2000; Aguilar-Aguilar \& Contreras, 2001; Contreras et al., 2001). En ese sentido, se ha propuesto a los NP como posibles áreas de conservación, ya que representan elementos taxonómicos con diversas historias biogeográficas (Grehan, 1989; 1993; Morrone \& EspinosaOrganista, 1998; Craw et al., 1999; Morrone, 2000; Aguilar-Aguilar \& Contreras, 2001; Contreras et al., 2001; Luna-Vega et al., 2010). Adicionalmente, los NP son importantes para la conservación al presentar endémicos locales, alta diversidad filogenética, tener afinidad geográfica compleja, y ser sitios que promueven la diversificación biótica (Heads, 1998; 2004; Escalante et al., 2004).

En la práctica, la priorización de los NP ha incluido los criterios de la riqueza de especies y complementariedad; esta última se refiere a representar mediante un sitio, las especies que no han sido reconocidas en una selección inicial (Vane-Wright et al., 1991). La complementariedad puede cuantificarse a través de índices (Colwell \& Coddington, 1994), cuyos valores van de 0 a 1 , donde el 0 indica coincidencia total en el número de especies que integran cada par de sitios, mientras que el valor 1 significa que hay complementariedad total, por lo tanto, ambos sitios no comparten especies.

El Componente Mexicano de Montaña fue descrito por Morrone y Márquez (2003) como un área geográfica donde se superponen las regiones Neártica y Neotropical; en dicha área habitan taxones espacialmente homólogos, cuya ubicación abarca cinco provincias biogeográficas: la Sierra Madre Occidental, la Sierra Madre Oriental, la Faja Volcánica Transmexicana, la Sierra Madre del Sur y las Tierras Altas de Chiapas (Morrone et al., 2017; Morrone, 2019). Uno de los grupos taxonómicos más utilizados como modelo para aproximarse a los estudios biogeográficos son los mamíferos, quienes además aportan numerosos servicios ambientales que generan bienestar social (Rojas \& Moreno, 2014), por ejemplo: (1) la producción de frutos comerciales derivados de plantas nocturnas que solo son polinizadas por murciélagos; (2) la reducción de insectos que son considerados plagas; y (3) el mantenimiento de suelos y 
bosques saludables por roedores y carnívoros. Así mismo, los mamíferos son un grupo con una importante connotación cultural en las regiones indígenas de México, donde son utilizados principalmente como alimento y medicina (Estrada-Portillo et al., 2018; Lorenzo et al., 2007; Barrasa, 2013; Monroy \& García-Flores, 2013; González \& Vallejo, 2014), contribuyendo a la canasta básica de grupos indígenas que presentan condiciones de pobreza y disponibilidad de recursos naturales (Bolkovic, 1997; Morales, 2000). Finalmente, los mamíferos también tienen importantes funciones sociales y recreativas (Pérez et al., 1995; Pérez, 1998; García, 2008).

Además del elemento evolutivo, las prácticas de conservación biológica deben considerar la relación de los pueblos indígenas con la naturaleza, la cual está basada en saberes ambientales mediados por sus costumbres; de esta manera los pueblos han mantenido una relación sustentable con la naturaleza, realizando actividades que permiten afrontar el deterioro ambiental (Boege, 2008; CDI, 2017). Por estas razones, los pueblos indígenas han sido reconocidos como sujetos sociales para la conservación de la biodiversidad y de los recursos naturales (Boege, 2008). Sin embargo, la representación de los pueblos indígenas en el espacio no resulta sencillo, ya que sus áreas de distribución son difusas y varían a lo largo del tiempo (Pérez et al., 2008). A pesar de esta problemática, la identificación de áreas geográficas donde habitan estos grupos indígenas ha sido objeto de estudio de algunos investigadores (Pérez et al., 2008). A la fecha, no existen análisis biogeográficos que incluyan esta información en la caracterización de los NP, con base en sus características culturales y la distribución de los pueblos indígenas.

Dado que México es uno de los países con una correlación estrecha entre su gran diversidad biológica y cultural, y con un gran número de aspectos culturales que tienen correspondencia con las áreas de mayor diversidad biológica (Sarukhán et al., 2009), el objetivo de esta investigación se centró en la priorización y caracterización ambiental y cultural de Nodos Panbiogeográficos (NP) para la conservación de mamíferos en el centro-sur de México.

\section{MATERIALES Y MÉTODOS}

Recientemente, Escalante et al. (2018) identificaron sitios de alta complejidad geológicatopográfica de América del Norte mediante NP de mamíferos, la mayoría reconocidos en zonas donde existe convergencia entre placas tectónicas. En México, las principales áreas nodo-diversas identificadas se localizan en el centro y sur del país, con 163 NP en el centro-sur de México (Escalante et al., 2018). Para el presente estudio, estos NP fueron superpuestos en el programa ArcMap 10.5 (ESRI, 2020), a las provincias biogeográficas que corresponden a la Faja Volcánica Transmexicana, Sierra Madre del Sur y Tierras Altas de Chiapas (Morrone et al., 2017). A partir de esta superposición, se reconoció un total de 84 NP, los cuales fueron enumerados de acuerdo con su latitud (de mayor a menor). Posteriormente se verificó cartográficamente la presencia espacial de las especies, mediante la presencia de los trazos individuales con los NP, dado que los trazos generalizados provienen de un proceso de superposición a partir de un buffer (Rojas-Parra, 2007; Escalante et al., 2017). Debido a este proceso, se omitieron los NP en los que: (1) ningún trazo individual se superpuso; y (2) en los que solo hubo superposición de trazos de especies de un único trazo generalizado. Así, únicamente $34 \mathrm{NP}$, que incluyeron a los trazos individuales de 35 especies, fueron seleccionados para continuar con la priorización. De los 34 NP seleccionados, 31 
se clasificaron en siete agrupamientos de NP sustentados por la convergencia de los mismos trazos generalizados, representando un mismo patrón biogeográfico, a los que se les llamó "Grupos". Adicionalmente, se reconocieron tres NP sustentados por convergencia de distintos trazos generalizados, los cuales no fueron agrupados y se trataron de manera independiente.

La riqueza de especies se estimó mediante el número de especies presentes en cada NP. Para priorizar los NP dentro de los grupos se siguieron los siguientes criterios: (1) a todos los grupos con más de dos NP (Grupos 1, 2 y 5), se les aplicó el Índice de Complementariedad (IC) de Colwell y Coddington (1994) mediante la fórmula: $I C=(a+b-2 j) /(a+b-j)$. Dónde: $a$ es el número de especies en el sitio 1 (cada sitio es un NP); $b$ es el número de especies en el sitio $2 ;$ y $j$ es el número de especies compartidas entre ambos sitios. (2) Para los cuatro grupos sustentados por un par de NP, ambos NP fueron seleccionados como prioritarios y se fusionaron en un solo NP, ya que tuvieron la misma composición biótica (Grupos 3, 4, 6 y 7). (3) Finalmente, los tres NP que no forman parte de ningún grupo fueron considerados prioritarios.

Los NP Prioritarios (NPP) de cada grupo fueron superpuestos con las provincias biogeográficas de Morrone et al. (2017) y con el marco cartográfico del Mapa Digital de México de INEGI (2017a), con el fin de ubicarlos a partir del punto cardinal más cercano de la característica geográfica (natural y/o social; por ejemplo: ríos, cuevas, cerros, cabeceras municipales, entre otros; INEGI, 2014d). Posteriormente, los NPP se superpusieron cartográficamente en QGIS Desktop 2.14.2 (QGIS Development Team, 2016) y en ArcGIS 10.1 (ESRI, 2012), con las capas digitales que representan las diferentes características ambientales, con el objetivo de caracterizar los NPP de una perspectiva físico-geográfica, de acuerdo con la propuesta de Álvarez-Mondragón y Morrone (2004) y García-Marmolejo et al. (2008). Dado que, como se mencionó anteriormente, los NP corresponden a la geometría de punto y carecen de dimensión espacial (Miguel-Talonia \& Escalante, 2013; Escalante et al., 2017), se realizó una intersección de puntos con polígonos, en la que se integraron los atributos de las coberturas geográficas a los NP (ESRI, 2020). Las capas digitales fueron seleccionadas para caracterizar física, geográfica y ambientalmente a los NP, de acuerdo con Álvarez-Mondragón y Morrone (2004) y García-Marmolejo et al. (2008). La cobertura de rocas fue obtenida de INEGI (2017b), mientras que las capas de hipsometría (INEGI et al., 1990), climas (García \& CONABIO, 1998), edafología (INEGI, 2014b), uso de suelo-vegetación (Rzedowski, 1978; Huber \& Riina, 2003; INEGI, 2017c), hidrografía (Maderey \& Torres-Ruata, 1990) y cuencas hidrográficas (INEGI et al., 2007), se obtuvieron del Portal de Geoinformación del Sistema Nacional de Información sobre la Biodiversidad (SNIB; Conabio, 2017).

El siguiente paso consistió en superponer los NPP con las áreas de distribución de los pueblos indígenas, con el fin de caracterizarlos culturalmente. Estas áreas fueron construidas con base en los datos del Atlas Nacional de los Pueblos Indígenas (CDI, 2018), reconociendo el nombre de los municipios en los que dichos pueblos se distribuyen. Para obtener espacialmente estas unidades político-administrativas, se utilizó la información digital de INEGI (2016a; b), del Portal de Geoinformación del Sistema Nacional de Información sobre la Biodiversidad (SNIB; Conabio, 2017). Así, las áreas de distribución de los pueblos indígenas se construyeron mediante una operación de unión de los municipios que comparten la presencia de un determinado pueblo en ArcMap 10.5 (ESRI, 2020). 


\section{RESULTADOS}

Los siete grupos que integraron a los $34 \mathrm{NP}$, así como las 35 especies involucradas en cada NP se describen en el Cuadro 1, siguiendo la nomenclatura taxonómica de Ramírez-Pulido et al. (2014), excepto para el género Oryzomys, que fue tratado aquí como Handleyomys, como sugieren Weksler et al. (2006) y Almendra et al. (2014). A partir de los criterios de priorización, 23 NP se clasificaron como prioritarios (NPP): (1) 12 NP mediante el IC, (2) 13 NP fusionados, y (3) tres nodos que no formaban parte de ningún grupo (Cuadro 1). En esos NPP, sólo cinco especies no fueron priorizadas (Handleyomys rostratus, Heteromys desmarestianus, Notocitellus adocetus, Peromyscus furvus y Sciurus deppei). La caracterización ambiental y cultural de los NPP se muestra en el Cuadro 2. La mayoría de los NPP se concentró en tres áreas geográficas: (1) oeste de la Faja Volcánica Transmexicana y Sierra Madre del Sur; (2) noreste de la Sierra Madre del Sur (Sierra Juárez) y (3) norte de las Tierras Altas de Chiapas. A continuación, se describe brevemente la localización de los NPP por cada grupo y su riqueza de especies.

Cuadro 1. Grupos de Nodos Panbiogeográficos (NP) en el centro-sur de México con las especies de mamíferos implicadas. Para cada NP se muestra si fue clasificado como prioritario (NPP); y para los NP de los Grupos 1, 2 y 5 se presenta el valor máximo del Índice de complementariedad (IC; Colwell \& Coddington, 1994). $S / G=$ Sin grupo.

\begin{tabular}{|c|c|c|c|c|}
\hline Grupo & NP & NPP & $\begin{array}{c}\text { Valor máximo } \\
\text { del IC }\end{array}$ & Especies \\
\hline \multirow[t]{5}{*}{1} & 2 & - & - & $\begin{array}{l}\text { Cryptotis alticola, Peromyscus hylocetes, } \\
\text { Reithrodontomys sumichrasti, Sciurus aureogaster, } \\
\text { Sorex mediopua }\end{array}$ \\
\hline & 3 & sí & 0.75 & $\begin{array}{l}\text { Cryptotis alticola, Peromyscus hylocetes, } \\
\text { Reithrodontomys sumichrasti, Sciurus aureogaster, } \\
\text { Sigmodon alleni, Sorex mediopua }\end{array}$ \\
\hline & 4 & sí & 0.75 & $\begin{array}{l}\text { Macrotus waterhousii, Peromyscus perfulvus, } \\
\text { Reithrodontomys sumichrasti, Sorex mediopua }\end{array}$ \\
\hline & 5 & sí & 0.75 & $\begin{array}{l}\text { Handleyomys melanotis, Peromyscus perfulvus, } \\
\text { Reithrodontomys sumichrasti, Sorex mediopua }\end{array}$ \\
\hline & 6 & - & - & $\begin{array}{l}\text { Notocitellus adocetus, Reithrodontomys sumichrasti, } \\
\text { Sciurus aureogaster, Sorex mediopua, Sylvilagus } \\
\text { cunicularius }\end{array}$ \\
\hline \multirow[t]{5}{*}{2} & 7 & - & - & $\begin{array}{l}\text { Handleyomys chapmani, Megadontomys cryophilus, } \\
\text { Peromyscus furvus, Peromyscus melanocarpus }\end{array}$ \\
\hline & 8 & sí & 1.00 & $\begin{array}{l}\text { Handleyomys chapmani, Megadontomys cryophilus, } \\
\text { Peromyscus beatae, Peromyscus melanocarpus }\end{array}$ \\
\hline & 10 & - & - & $\begin{array}{l}\text { Cryptotis mexicanus, Megadontomys cryophilus, } \\
\text { Peromyscus furvus }\end{array}$ \\
\hline & 11 & - & - & $\begin{array}{l}\text { Cryptotis mexicanus, Heteromys desmarestianus, } \\
\text { Megadontomys cryophilus }\end{array}$ \\
\hline & 12 & - & - & $\begin{array}{l}\text { Handleyomys chapmani, Megadontomys cryophilus, } \\
\text { Peromyscus melanocarpus }\end{array}$ \\
\hline
\end{tabular}




\begin{tabular}{|c|c|c|c|c|}
\hline Grupo & NP & NPP & $\begin{array}{l}\text { Valor máximo } \\
\text { del IC }\end{array}$ & Especies \\
\hline & 15 & - & - & $\begin{array}{l}\text { Handleyomys chapmani, Handleyomys rostratus, } \\
\text { Megadontomys cryophilus, Peromyscus melanocarpus }\end{array}$ \\
\hline & 16 & - & - & $\begin{array}{l}\text { Cryptotis mexicanus, Handleyomys chapmani, } \\
\text { Megadontomys cryophilus, Peromyscus melanocarpus }\end{array}$ \\
\hline & 17 & sí & 1.00 & Cryptotis mexicanus, Megadontomys cryophilus \\
\hline & 18 & - & - & $\begin{array}{l}\text { Handleyomys chapmani, Megadontomys cryophilus, } \\
\text { Peromyscus melanocarpus, Sciurus deppei }\end{array}$ \\
\hline & 19 & - & - & Megadontomys cryophilus, Sciurus deppei \\
\hline & 22 & - & - & $\begin{array}{l}\text { Handleyomys chapmani, Handleyomys rostratus, } \\
\text { Megadontomys cryophilus, Peromyscus melanocarpus }\end{array}$ \\
\hline \multirow[t]{2}{*}{3} & 9 & Sí & - & $\begin{array}{l}\text { Cryptotis mexicanus, Handleyomys chapmani, } \\
\text { Megadontomys cryophilus, Peromyscus melanocarpus }\end{array}$ \\
\hline & 13 & Sí & - & $\begin{array}{l}\text { Handleyomys chapmani, Megadontomys cryophilus, } \\
\text { Peromyscus melanocarpus }\end{array}$ \\
\hline \multirow[t]{2}{*}{4} & 14 & sí & - & Megadontomys cryophilus, Sciurus aureogaster \\
\hline & 21 & Sí & - & Megadontomys cryophilus, Sciurus aureogaster \\
\hline \multirow[t]{7}{*}{5} & 23 & Sí & 1.00 & $\begin{array}{l}\text { Peromyscus zarhynchus, Reithrodontomys sumichrasti, } \\
\text { Sciurus aureogaster, Tylomys tumbalensis }\end{array}$ \\
\hline & 28 & sí & 1.00 & Handleyomys rhabdops, Sciurus aureogaster \\
\hline & 30 & Sí & 1.00 & $\begin{array}{l}\text { Peromyscus guatemalensis, Peromyscus zarhynchus, } \\
\text { Reithrodontomys sumichrasti, Sciurus aureogaster }\end{array}$ \\
\hline & 31 & Sí & 1.00 & Peromyscus guatemalensis, Sciurus aureogaster \\
\hline & 32 & Sí & 1.00 & $\begin{array}{l}\text { Handleyomys rhabdops, Peromyscus zarhynchus, } \\
\text { Reithrodontomys sumichrasti, Sciurus aureogaster }\end{array}$ \\
\hline & 34 & Sí & 1.00 & $\begin{array}{l}\text { Peromyscus guatemalensis, Reithrodontomys } \\
\text { sumichrasti, Reithrodontomys tenuirostris, Sciurus } \\
\text { aureogaster }\end{array}$ \\
\hline & 35 & Sí & 1.00 & Heteromys nelsoni, Myotis fortidens \\
\hline \multirow[t]{2}{*}{6} & 24 & Sí & - & Pteronotus gymnonotus, Tylomys nudicaudus \\
\hline & 25 & Sí & - & Pteronotus gymnonotus, Reithrodontomys sumichrasti \\
\hline \multirow[t]{2}{*}{7} & 44 & Sí & - & Myotis fortidens, Pteronotus gymnonotus \\
\hline & 46 & sí & - & Baiomys musculus, Pteronotus gymnonotus \\
\hline \multirow[t]{3}{*}{$S / G$} & 1 & Sí & - & $\begin{array}{l}\text { Peromyscus hylocetes, Sciurus colliaei, Sciurus } \\
\text { nayaritensis, Sigmodon mascotensis, Sorex mediopua, } \\
\text { Sylvilagus cunicularius }\end{array}$ \\
\hline & 26 & Sí & - & Cryptotis mexicanus, Handleyomys rhabdops \\
\hline & 33 & Sí & - & $\begin{array}{l}\text { Orthogeomys grandis, Reithrodontomys sumichrasti, } \\
\text { Sciurus aureogaster, Sigmodon mascotensis, Sorex } \\
\text { veraepacis }\end{array}$ \\
\hline
\end{tabular}


Cuadro 2. Características ambientales y culturales de los Nodos Panbiogeográficos Prioritarios (NPP). GPO= Grupo, NPP = Nodo Panbiogeográfico Prioritario, NPPF= Nodos Panbiogeográficos Prioritarios Fusionados, $\mathrm{S} / \mathrm{G}=$ Sin grupo.

\begin{tabular}{|c|c|c|c|c|c|c|c|c|}
\hline 운 & $\frac{a}{2}$ & $\begin{array}{l}\mathscr{J} \\
\mathscr{x} \\
\mathscr{x}\end{array}$ & 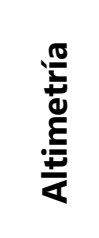 & $\stackrel{\text { E⿱ }}{\bar{U}}$ & $\frac{0}{\frac{0}{\omega}}$ & 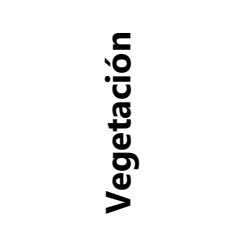 & 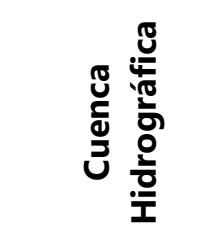 & $\begin{array}{l}: 0 \frac{\pi}{0} \\
\frac{0}{0} \\
\frac{\pi}{0} \\
\frac{0}{0} \\
\frac{0}{0}\end{array}$ \\
\hline \multirow[t]{3}{*}{1} & 3 & \multirow[t]{3}{*}{$\begin{array}{l}\text { Ígnea } \\
\text { extrusiva }\end{array}$} & 3700 & $\mathrm{E}(\mathrm{T}) \mathrm{CHw}$ & \multirow[t]{3}{*}{ Regosol } & $\begin{array}{l}\text { Bosque de } \\
\text { Pino }\end{array}$ & Coahuayana & \multirow[t]{3}{*}{-} \\
\hline & 4 & & 1600 & $(A) C(w 2)$ & & $\begin{array}{l}\text { Bosque de } \\
\text { Pino-Encino }\end{array}$ & Armería & \\
\hline & 5 & & 1900 & & & $\begin{array}{l}\text { Bosque de } \\
\text { Pino-Encino }\end{array}$ & & \\
\hline \multirow[t]{2}{*}{2} & 8 & $\begin{array}{l}\text { Ígnea } \\
\text { extrusiva }\end{array}$ & 2300 & \multirow[t]{2}{*}{$C(m)$} & Luvisol & $\begin{array}{l}\text { Bosque de } \\
\text { Pino-Encino }\end{array}$ & \multirow[t]{2}{*}{ Papaloapan } & $\begin{array}{l}\text { Cuicatecos y } \\
\text { Mazatecos }\end{array}$ \\
\hline & 17 & Esquisto & 1700 & & Acrisol & $\begin{array}{l}\text { Bosque } \\
\text { Mesófilo de } \\
\text { Montaña }\end{array}$ & & Zapotecos \\
\hline 3 & NPPF & Esquisto & $\begin{array}{l}800- \\
3000\end{array}$ & $\begin{array}{l}C(m), \\
(A) C(m)(f) \\
\text { y }(A) C(m)\end{array}$ & $\begin{array}{l}\text { Acrisol, } \\
\text { posiblem- } \\
\text { ente } \\
\text { cambisol }\end{array}$ & $\begin{array}{l}\text { Bosque } \\
\text { Mesófilo de } \\
\text { Montaña, } \\
\text { Bosque de } \\
\text { Pino-Encino, } \\
\text { Bosque de } \\
\text { Pino y } \\
\text { Bosque } \\
\text { lluvioso } \\
\end{array}$ & Papaloapan & $\begin{array}{l}\text { Zapotecos y } \\
\text { Chinantecos }\end{array}$ \\
\hline 4 & NPPF & $\begin{array}{l}\text { Esquisto, } \\
\text { Caliza, } \\
\text { Ígnea- } \\
\text { extrusiva, } \\
\text { Lutita y } \\
\text { Pizarra }\end{array}$ & $\begin{array}{l}1700 \\
- \\
3000\end{array}$ & $\begin{array}{l}C(m), \\
C(w 2), \\
A m, C(w 1) \\
\text { y } C b^{\prime}(m)\end{array}$ & $\begin{array}{l}\text { Acrisol y } \\
\text { Luvisol }\end{array}$ & $\begin{array}{l}\text { Bosque de } \\
\text { Pino, Bosque } \\
\text { de Pino- } \\
\text { Encino, } \\
\text { Bosque } \\
\text { Mesófilo de } \\
\text { Montaña y } \\
\text { Agricultura } \\
\text { de Temporal }\end{array}$ & Papaloapan & Zapotecos \\
\hline \multirow[t]{5}{*}{5} & 23 & Caliza & 1200 & $(\mathrm{~A}) \mathrm{C}(\mathrm{fm})$ & Leptosol & $\begin{array}{l}\text { Pastizal } \\
\text { cultivado }\end{array}$ & \multirow[t]{5}{*}{$\begin{array}{l}\text { Grijalva- } \\
\text { Usumacinta }\end{array}$} & Choles \\
\hline & 28 & $\begin{array}{l}\text { Limolita- } \\
\text { Arenisca }\end{array}$ & 1700 & $(A) C(m)(f)$ & \multirow[t]{2}{*}{ Luvisol } & $\begin{array}{l}\text { Bosque de } \\
\text { Pino }\end{array}$ & & \multirow[t]{3}{*}{-} \\
\hline & 30 & $\begin{array}{l}\text { Ígnea } \\
\text { extrusiva }\end{array}$ & 2600 & $\mathrm{Cb}^{\prime}(\mathrm{w} 2)$ & & $\begin{array}{l}\text { Bosque de } \\
\text { Pino-Encino }\end{array}$ & & \\
\hline & 31 & Caliza & 2200 & $C(w 2)$ & Gleysol & $\begin{array}{l}\text { Bosque de } \\
\text { Encino }\end{array}$ & & \\
\hline & 32 & & & & & $\begin{array}{l}\text { Zonas } \\
\text { Urbanas }\end{array}$ & & - \\
\hline
\end{tabular}




\begin{tabular}{|c|c|c|c|c|c|c|c|c|c|}
\hline 운 & $\frac{n}{z}$ & 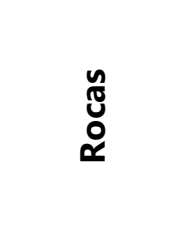 & 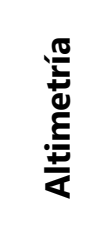 & 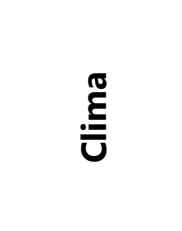 & $\frac{0}{\stackrel{0}{\nu}}$ & 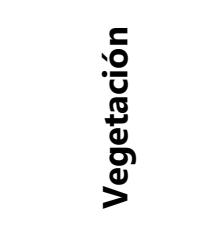 & ฮ & 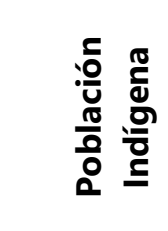 & \\
\hline & 34 & $\begin{array}{l}\text { Limolita- } \\
\text { Arenisca }\end{array}$ & 2650 & $C(m)$ & Acrisol & $\begin{array}{l}\text { Bosque } \\
\text { Mesófilo de } \\
\text { Montaña }\end{array}$ & Huehuetan & $\begin{array}{l}\text { Mames } \\
\text { Mochos }\end{array}$ & y \\
\hline & 35 & $\begin{array}{l}\text { Ígnea } \\
\text { intrusiva }\end{array}$ & 2300 & & & $\begin{array}{l}\text { Agricultura } \\
\text { de Temporal }\end{array}$ & & Mames & \\
\hline 6 & NPPF & $\begin{array}{l}\text { Calíza, } \\
\text { Lutita- } \\
\text { Arenisca, } \\
\text { Ígnea } \\
\text { extrusiva, } \\
\text { Limolita- } \\
\text { Arenisca }\end{array}$ & $\begin{array}{l}600- \\
2400\end{array}$ & $\begin{array}{l}A f, \\
C(m)(f), \\
(A) C(f m), \\
C(f), A w 2 \text { y } \\
(A) C(m)(f)\end{array}$ & $\begin{array}{l}\text { Leptosol, } \\
\text { Luvisol, } \\
\text { Acrisol, } \\
\text { Rendzina } \\
\text { y Feozem }\end{array}$ & $\begin{array}{l}\text { Pastizal } \\
\text { cultivado, } \\
\text { Bosque } \\
\text { Mesófilo de } \\
\text { Montaña, } \\
\text { Bosque } \\
\text { lluvioso } \\
\text { tropical, } \\
\text { Bosque de } \\
\text { Pino-Encino, } \\
\text { Bosque de } \\
\text { Pino y Zonas } \\
\text { Urbanas }\end{array}$ & $\begin{array}{l}\text { Grijalva- } \\
\text { Usumacinta }\end{array}$ & - & \\
\hline 7 & NPPF & $\begin{array}{l}\text { Caliza, } \\
\text { posibleme } \\
\text { nte Lutita- } \\
\text { Arenisca y } \\
\text { Limolita- } \\
\text { Arenisca }\end{array}$ & $\begin{array}{l}400- \\
1800\end{array}$ & $\begin{array}{l}(A) C(m)(f), \\
\text { Am(f) y } \\
\text { posiblem- } \\
\text { ente } \\
\text { Aw2 }\left(x^{\prime}\right)\end{array}$ & $\begin{array}{l}\text { Luvisol y } \\
\text { Feozem }\end{array}$ & $\begin{array}{l}\text { Bosque de } \\
\text { Pino-Encino, } \\
\text { Bosque } \\
\text { lluvioso } \\
\text { tropical, } \\
\text { Bosque } \\
\text { Mesófilo de } \\
\text { Montaña y } \\
\text { Zonas } \\
\text { urbanas }\end{array}$ & $\begin{array}{l}\text { Grijalva- } \\
\text { Usumacinta }\end{array}$ & Tzotziles & \\
\hline \multirow[t]{3}{*}{$S / G$} & 1 & $\begin{array}{l}\text { Ígnea } \\
\text { extrusiva }\end{array}$ & 2200 & $C(w 2)$ & Regosol & \multirow[t]{3}{*}{$\begin{array}{l}\text { Bosque de } \\
\text { Pino-Encino }\end{array}$} & San Nicolás & - & \\
\hline & 26 & $\begin{array}{l}\text { Limolita- } \\
\text { Arenisca }\end{array}$ & 1150 & Aw2 & Leptosol & & $\begin{array}{l}\text { Grijalva- } \\
\text { Usumacinta }\end{array}$ & - & \\
\hline & 33 & Gneis & 2150 & $C(w 2)$ & Acrisol & & Copalita & Zapotecos & \\
\hline
\end{tabular}

Grupo 1: se localiza al oeste de la Faja Volcánica Transmexicana (Fig. 1); está conformado por cinco NP sustentados por 11 especies. Tres de los cinco NP fueron seleccionados como NPP con base en el IC; de ellos, el NP 3 se ubica en el Nevado de Colima (INEGI, 2014a), y los NP 4 y 5 en el Volcán de Colima (INEGI, 2014d).

Grupo 2: se localiza al este de la Sierra Madre del Sur (norte de Oaxaca, sobre Cuicatlán y la Sierra Juárez; Fig. 2). Este grupo está conformado por 11 NP sustentados por nueve especies. De los 11 
NP, dos fueron seleccionados como NPP con base en su IC; de ellos, el NP 8 se ubica al noreste de la cabecera municipal Santa María Pápalo (INEGI, 2014c) y el NP 17 se ubica al norte de la localidad La Luz (INEGI, 2001d).

Grupo 3: se ubica al norte de la Sierra Juárez (Fig. 3). Este grupo está conformado por dos NP fusionados y prioritarios, sustentados por cuatro especies. El NP 9 se ubica al noreste de la localidad Microondas y el NP 13 se ubica al sureste del cerro La cueva de Sarmiento (INEGI, 2001d).

Grupo 4: este grupo está localizado al suroeste de la Sierra Juárez (Fig. 4), y está conformado por dos NP fusionados y prioritarios, sustentados por dos especies. El NP 14 se ubica en el cerro Humo Grande y el NP 21 se ubica al este de la cabecera municipal San Juan Evangelista Analco (INEGI, 2001d). Este NPP fusionado se superpone con el NPP fusionado del Grupo 3.

Grupo 5: ubicado sobre las Tierras Altas de Chiapas (Fig. 5), este grupo está conformado por siete NP sustentados por nueve especies. Con base en los valores del IC, todos los NP de este grupo fueron seleccionados como NPP. EI NP 23 se ubica al sureste de la cabecera municipal de Tumbala (INEGI, 2004c), el NP 28 se ubica al sur de la localidad Las Maravillas (INEGI, 2004b), el NP 30 se ubica en la localidad Huitepec Ocotal Segunda Sección (INEGI, 2000), los NP 31 y 32 se encuentran al este de la cabecera municipal San Cristóbal de las Casas (INEGI, 2001c), el NP 34 se ubica al sureste de la localidad Mozotal (INEGI, 2002) y el NP 35 se localiza al oeste de El Pizarrín (INEGI, 2001b).

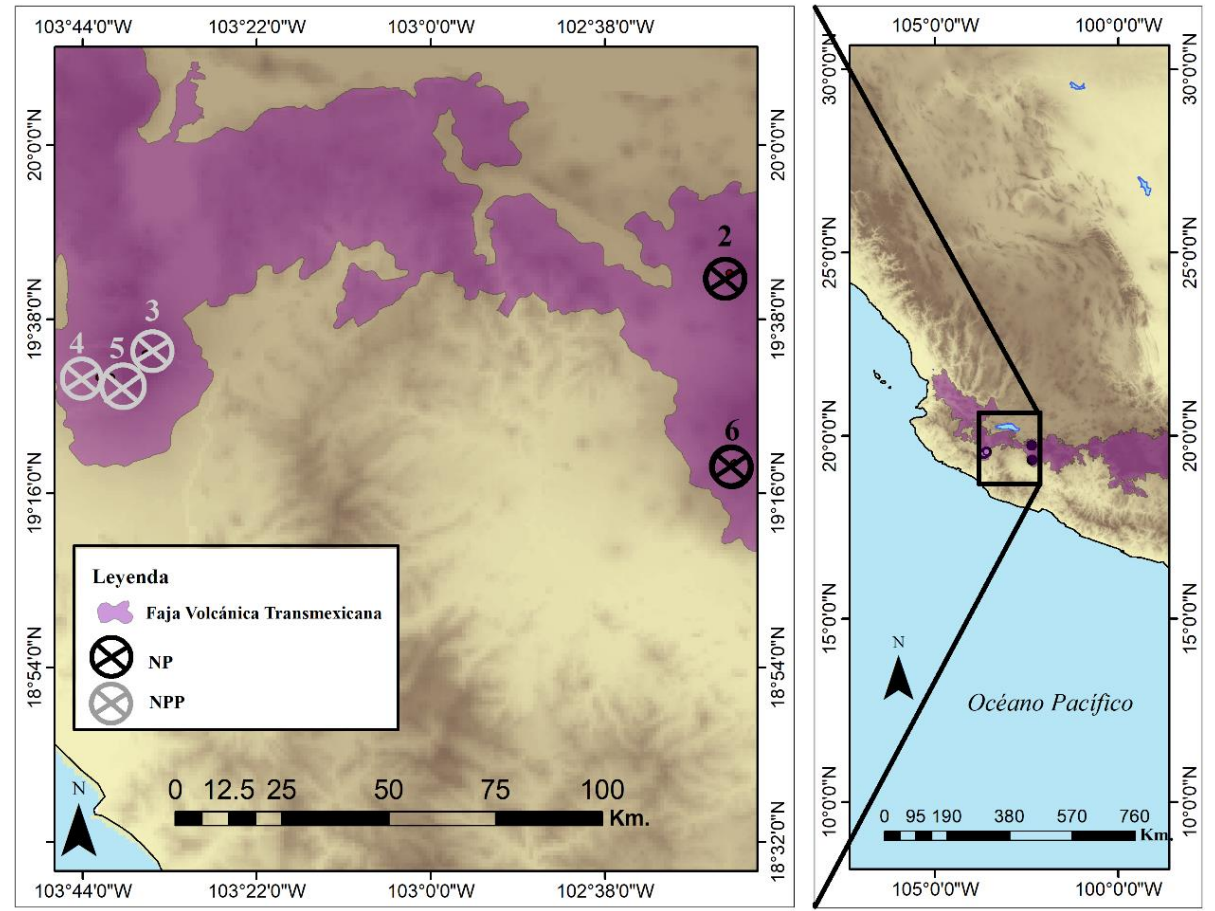

Figura 1. Nodos panbiogeográficos del Grupo 1. NP= Nodo Panbiogeográfico. NPP= Nodo Panbiogeográfico Prioritario. Elaborado con base en: EROS (2010), INEGI (2018), Morrone et al. (2017) y Natural Earth (2017a, b, c). 


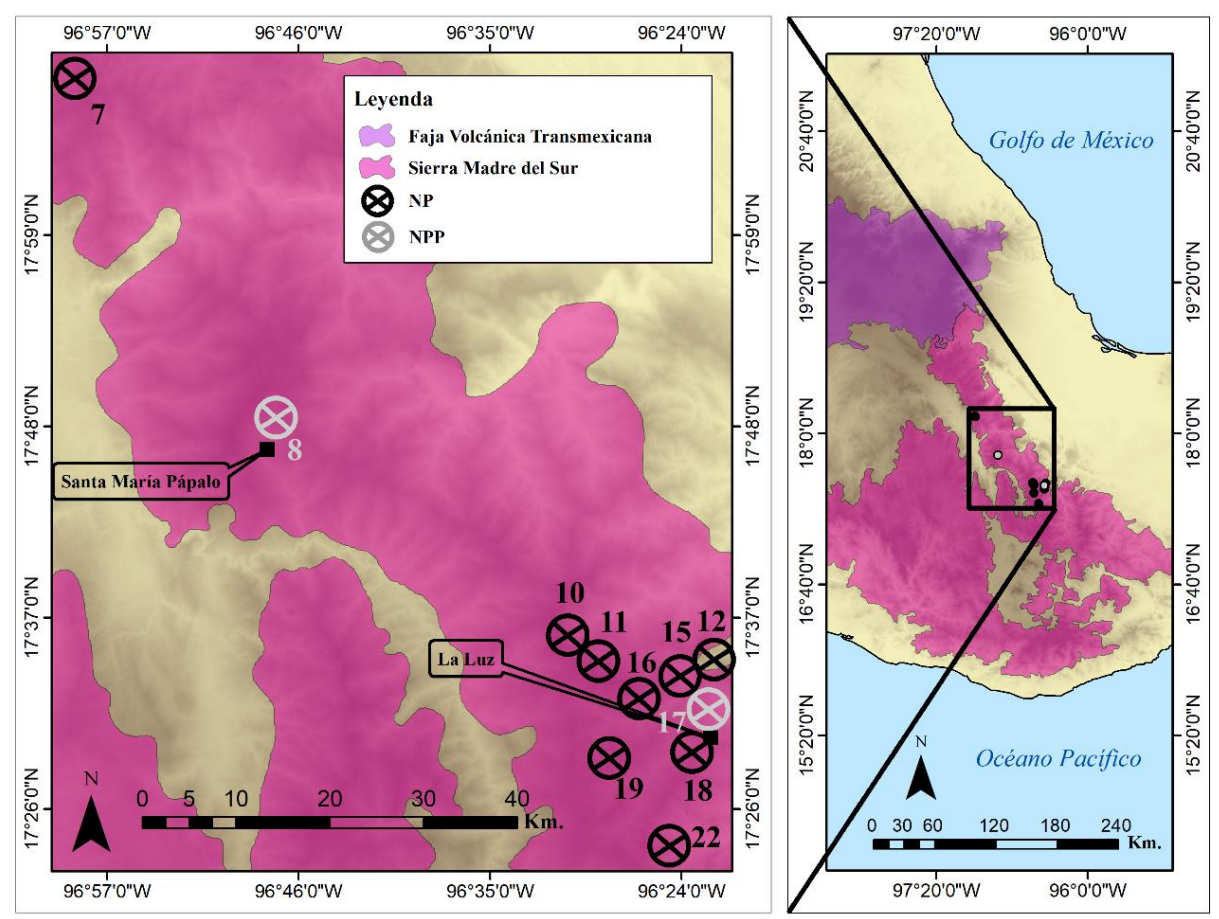

Figura 2. Nodos panbiogeográficos del Grupo 2. NP= Nodo Panbiogeográfico. NPP= Nodo Panbiogeográfico Prioritario. Elaborado con base en: EROS (2010), INEGI (2018), Morrone et al. (2017) y Natural Earth $(2017 a, b, c)$.
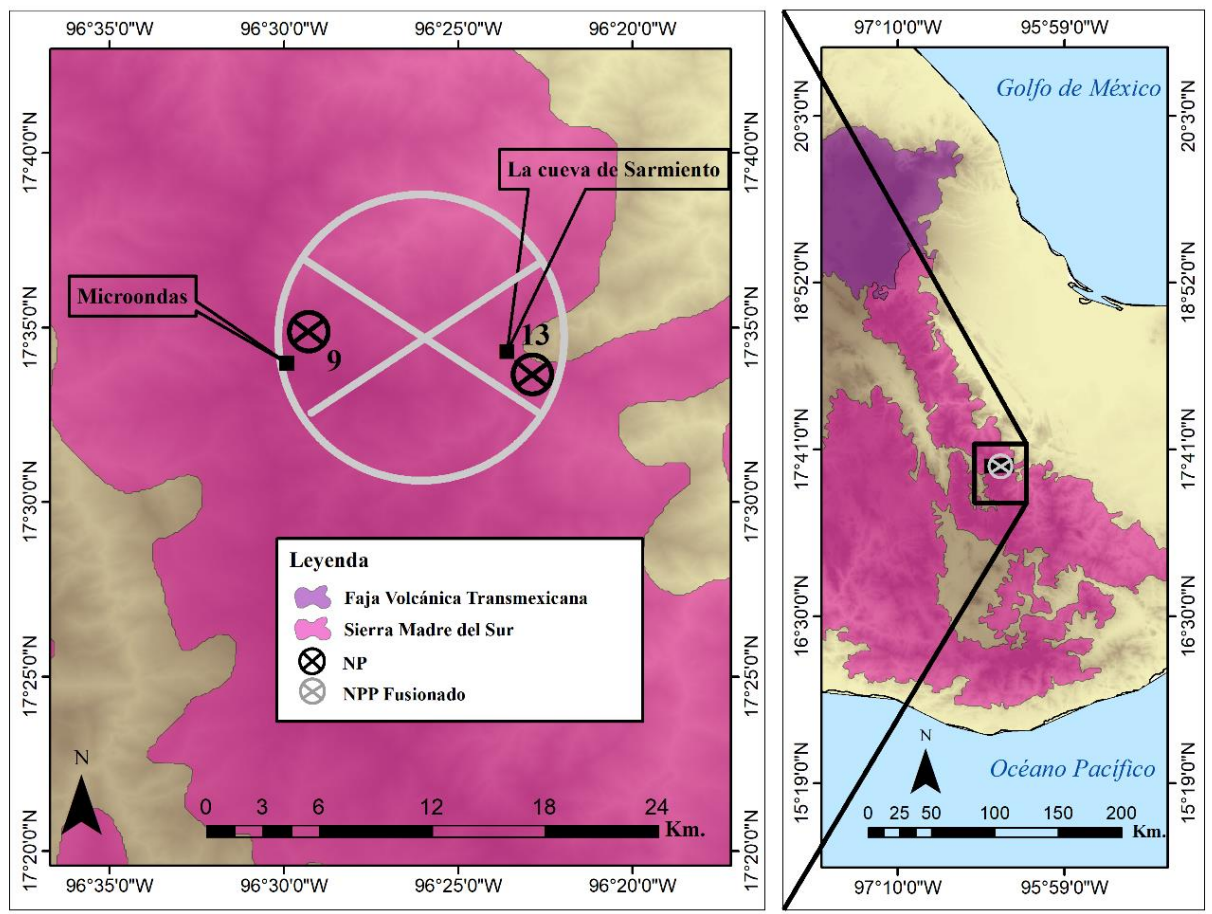

Figura 3. Nodos panbiogeográficos del Grupo 3. NP= Nodo Panbiogeográfico. NPP Fusionado= Nodo Panbiogeográfico Prioritario Fusionado. Elaborado con base en: EROS (2010), INEGI (2018), Morrone et al. (2017) y Natural Earth (2017a, b, c). 

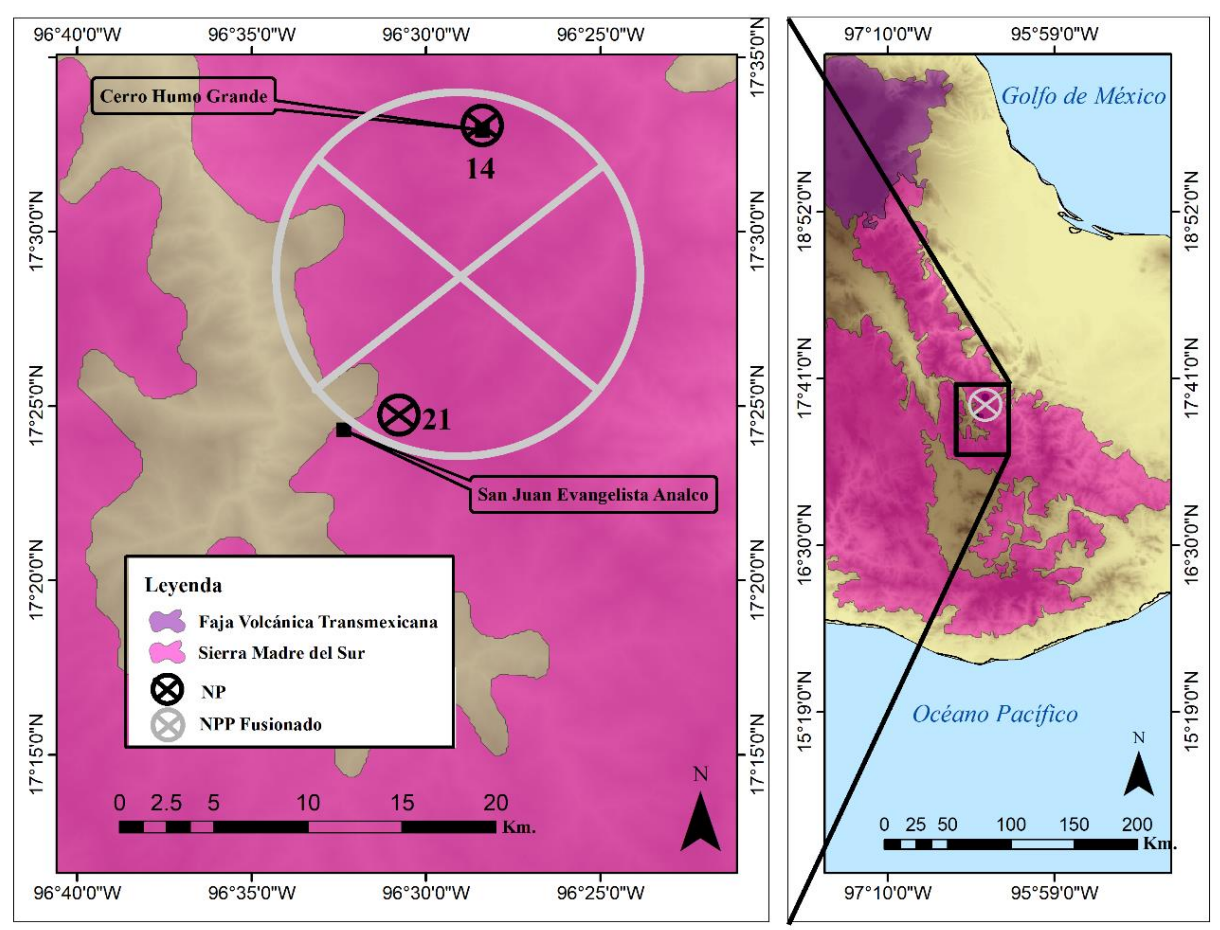

Figura 4. Nodos panbiogeográficos del Grupo 4. NP= Nodo Panbiogeográfico. NPP Fusionado = Nodo Panbiogeográfico Prioritario Fusionado. Elaborado con base en: EROS (2010), INEGI (2018), Morrone et al. (2017) y Natural Earth $(2017 a, b, c)$.
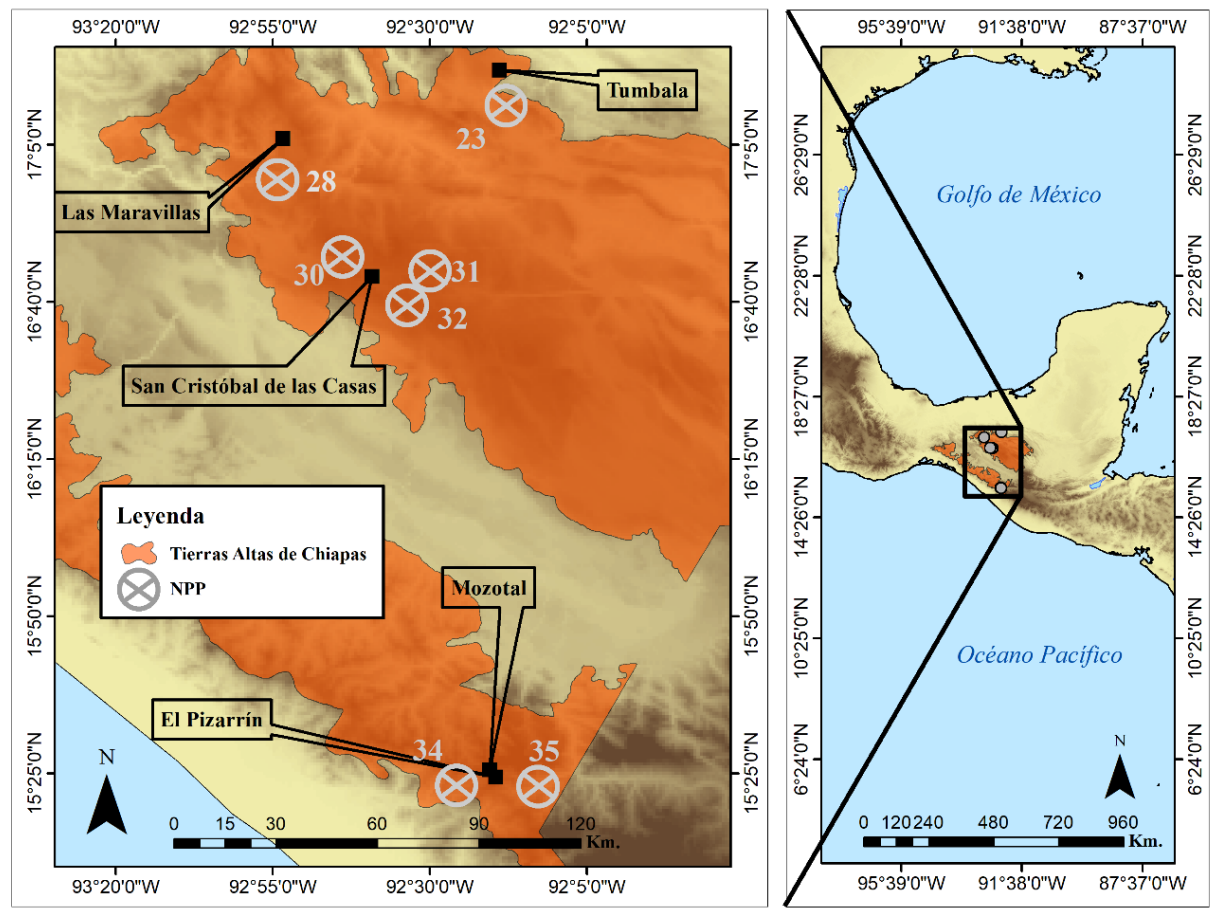

Figura 5. Nodos panbiogeográficos del Grupo 5. NP= Nodo Panbiogeográfico. NPP= Nodo Panbiogeográfico Prioritario. Elaborado con base en: EROS (2010), INEGI (2018), Morrone et al. (2017) y Natural Earth $(2017 a, b, c)$. 

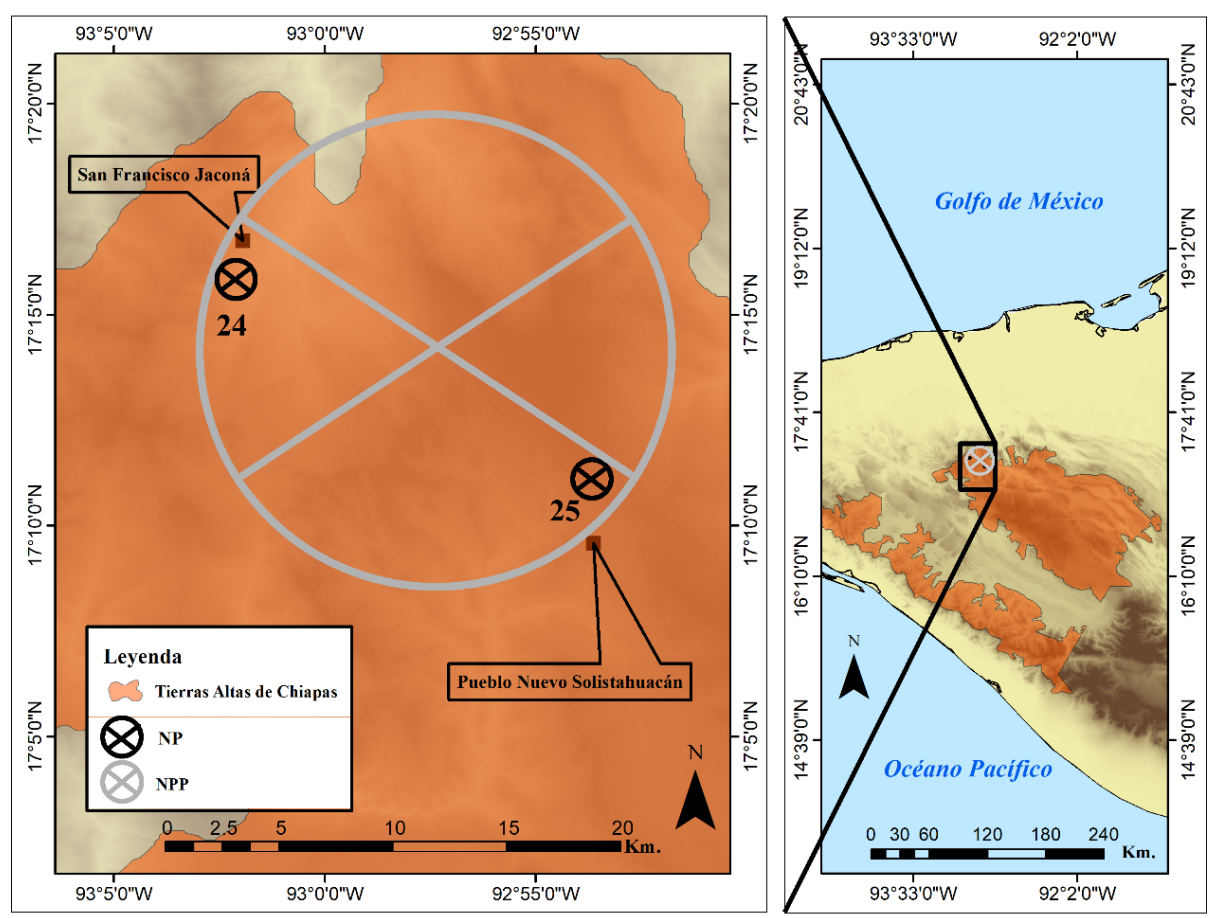

Figura 6. Nodos panbiogeográficos del Grupo 6. NP= Nodo Panbiogeográfico. NPP Fusionado = Nodo Panbiogeográfico Prioritario Fusionado. Elaborado con base en: EROS (2010), INEGI (2018), Morrone et al. (2017) y Natural Earth $(2017 a, b, c)$.
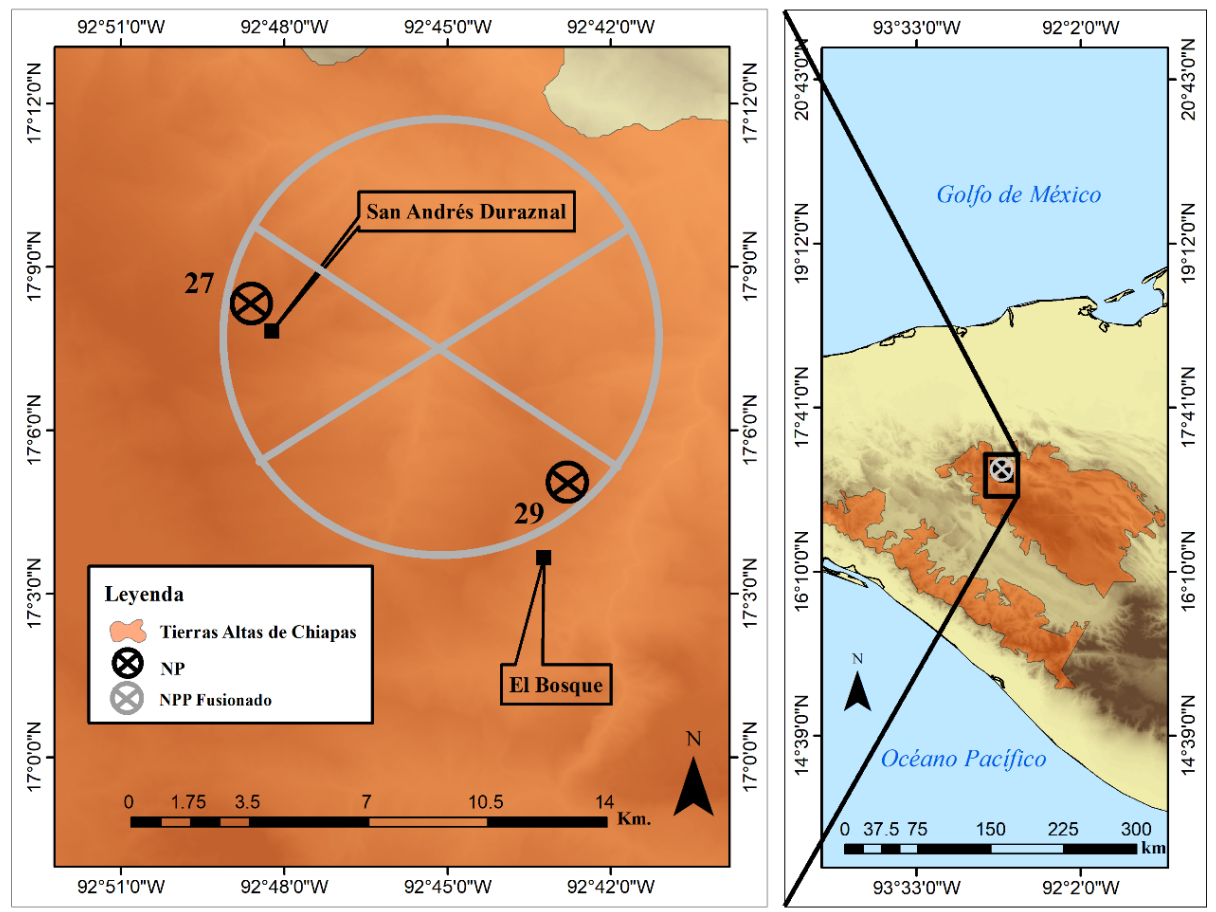

Figura 7. Nodos panbiogeográficos del Grupo 7. NP= Nodo Panbiogeográfico. NPP Fusionado = Nodo Panbiogeográfico Prioritario Fusionado. Elaborado con base en: EROS (2010), INEGI (2018), Morrone et al. (2017) y Natural Earth (2017a, b, c). 
Grupo 6: se localiza al noroeste de las Tierras Altas de Chiapas (Fig. 6). Este grupo está conformado por dos NP fusionados y prioritarios, sustentados por tres especies. El NP 24 se ubica al sur de la localidad de San Francisco Jaconá (INEGI, 2004a), y el NP 25 se localiza al norte de la cabecera municipal Pueblo Nuevo Solistahuacán (INEGI, 2004b).

Grupo 7: está ubicado al noroeste de las Tierras Altas de Chiapas (Fig. 7) y al sureste del Grupo 6. Este grupo está conformado por dos NP fusionados y prioritarios, sustentados por tres especies. El NP 27 se ubica al noroeste de la cabecera municipal San Andrés Duraznal y el NP 29 al norte de la cabecera municipal El Bosque (INEGI, 2004b).

Finalmente, los tres NPP sin grupo estuvieron sustentados por 12 especies (Fig. 8). El NP 1 se ubica al sureste de la localidad La Cumbre de Guadalupe [La Cumbre de los Arrastrados] (INEGI, 2001a), el NP 26 se encuentra al noroeste del cerro El Terrero (INEGI, 2004b) y, finalmente, el NP 33 se localiza al oeste de la localidad Río San José (INEGI, 2003).

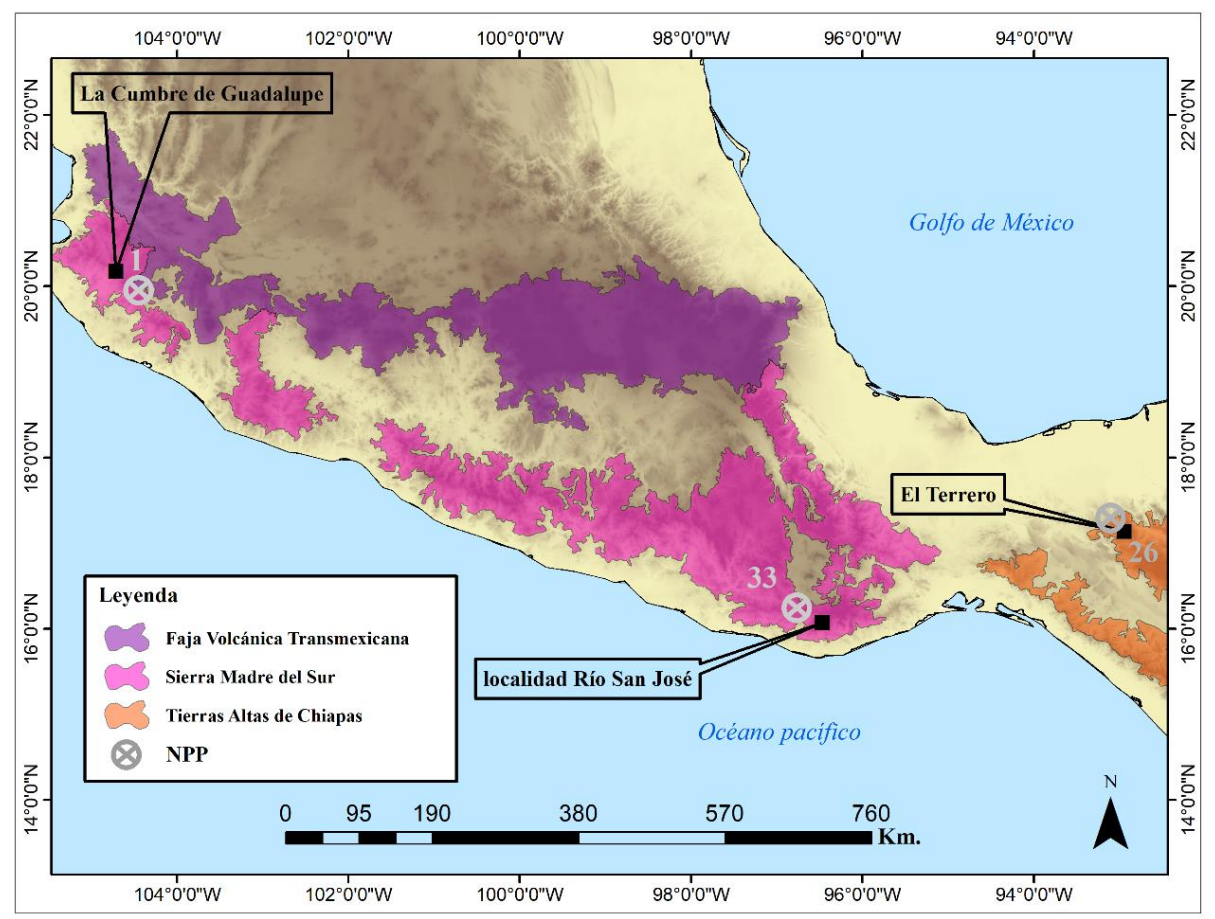

Figura 8. Nodos panbiogeográficos heterogéneos. NPP= Nodo Panbiogeográfico Prioritario. Elaborado con base en: EROS (2010), INEGI (2018), Morrone et al. (2017) y Natural Earth (2017a, b, c).

En general, se observó que la mayoría de los NPP fueron reconocidos sobre la provincia de las Tierras Altas de Chiapas, seguida por la Sierra Madre del Sur, siendo la Faja Volcánica Transmexicana la provincia con menor número de NP. En las dos primeras provincias existió superposición y aglomeración de NPP, específicamente sobre los Altos de Chiapas y la Sierra Juárez. Con respecto a la caracterización cultural, los principales grupos indígenas representados en los NPP fueron Chinantecos, Cuicatecos, Mazatecos y Zapotecos sobre la Sierra Juárez; 
Zapotecos en la parte meridional de la Sierra Madre del Sur; Choles y Tzotziles en los Altos de Chiapas; y Mames y Mochos en el Soconusco (Cuadro 2; Fig. 9).

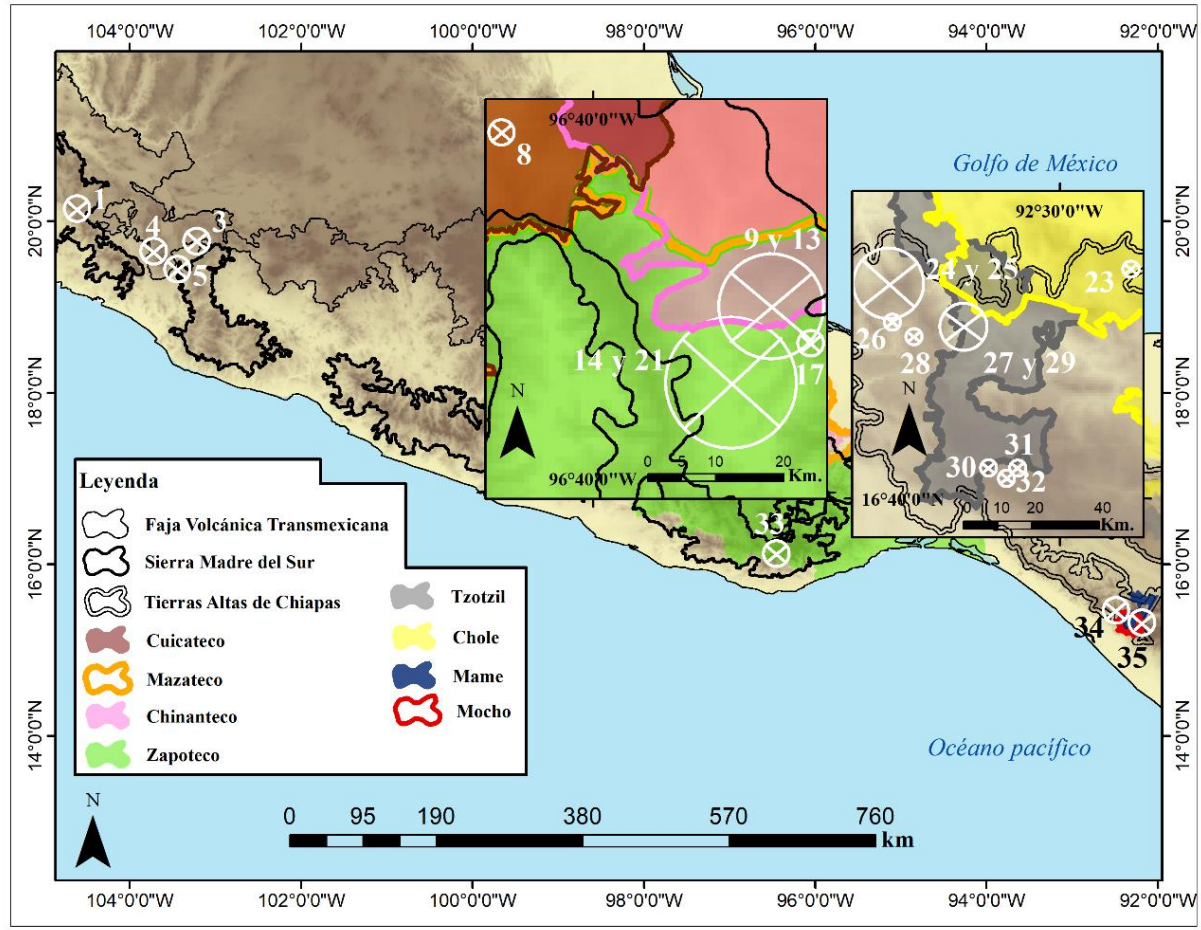

Figura 9. Nodos panbiogeográficos prioritarios las provincias biogeográficas del centro-sur de México; y distribución de pueblos indígenas. NPP = Nodo Panbiogeográfico Prioritario. Elaborado con base en: EROS (2010), INEGI (2018), Morrone et al. (2017) y Natural Earth (2017a, b, c).

\section{DISCUSIÓN}

Pocas áreas geográficas como México poseen una constitución tan compleja en sus elementos físico-geográficos, lo que dificulta su análisis. Así mismo, México cuenta con una gran diversidad biológica y cultural (Sarukhán et al., 2009; Semarnat-Instituto Nacional de Lenguas Indígenas, 2012). Por lo anterior, es necesaria la caracterización ambiental y cultural de los sitios más complejos geobióticamente representados mediante los NP de los mamíferos, los cuales podrán ser un punto de partida para describir el escenario para su conservación.

En los Altos de Chiapas se identificaron ocho NPP (algunos de ellos superpuestos), confirmando que es la zona geobióticamente más compleja del centro y sur de México, y posiblemente la zona más importante en términos de conservación, lo cual coincide con los hallazgos de Ochoa et al. (2003). También la Sierra Juárez presentó aglomeración y superposición de NPP, por lo que podría constituir la segunda zona más importante a ser conservada debido a su complejidad biótica. Otras áreas geográficas importantes son: Cuicatlán, la zona meridional de la Sierra Madre del Sur (Miahuatlán), y el Soconusco. 
Los NPP identificados al oeste de la Faja Volcánica Transmexicana coinciden de manera general con los NP de los trabajos de Escalante (2001), Torres y Luna-Vega (2006), GarcíaMarmolejo et al. (2008) y González-Ávila et al. (2017), donde el NP 8 se reconoce como prioritario por estos últimos autores. Los NPP sobre Cuicatlán y la Sierra Juárez coinciden con los NP reconocidos en los trabajos de Escalante (2001), Márquez y Morrone (2003), Álvarez-Mondragón y Morrone (2004), Escalante et al. (2004), García-Marmolejo et al. (2008) y Yañez-Ordoñez et al. (2008); siendo prioritarios también para García-Marmolejo et al. (2008). El NPP de la parte meridional de la Sierra Madre del Sur coincide con los NP identificados por Escalante (2001), Yañez-Ordoñez et al. (2008) y un NP prioritario de Ochoa et al. (2003). Los NPP reconocidos en los Altos de Chiapas coinciden con los NP identificados en los trabajos de Escalante (2001), Ochoa et al. (2003), Escalante et al. (2004), Corona y Morrone (2005), Corona y Toledo (2006), GarcíaMarmolejo et al. (2008), Yañez-Ordoñez et al. (2008) y González-Ávila et al. (2017), siendo prioritarios para Ochoa et al. (2003), García-Marmolejo et al. (2008) y González-Ávila et al. (2017). Finalmente, los NPP reconocidos en el Soconusco coinciden con los trabajos de ÁlvarezMondragón y Morrone (2004) y Yañez-Ordoñez et al. (2008).

Los taxones que han sido registrados en los NP de los diversos estudios incluyen aves, mamíferos, herpetofauna, insectos y hongos, siendo este último el taxón con menor cantidad de estudios biogeográficos (González-Ávila et al., 2017). Esto sugiere que al proponer como prioritarios los NPP para la conservación de los mamíferos, también se estarán preservando otros grupos taxonómicos. Sin embargo, los datos de las colecciones científicas de donde provienen los trazos individuales pueden presentar sesgos. Los principales sesgos de los datos de la biodiversidad que afectan a los estudios biogeográficos son aquellos relacionados con la cantidad de descripciones biológicas (sesgo Lineano) y de la falta de completitud del conocimiento geográfico (sesgo Wallaceano; Hortal et al., 2015). Esto es particularmente relevante al establecer estrategias de conservación, pero en la mayoría de los casos, los sesgos no son cuantificados (Oliveira et al., 2016). Por ejemplo, para los mamíferos se ha demostrado que las áreas más ricas en especies son también las más conocidas, incluyendo a los estados de Oaxaca y Chiapas, donde se ubican muchos de los NPP, y algunos otros estados que forman parte de la Faja Volcánica Transmexicana, como Jalisco y Michoacán (Ramírez-Pulido et al., 2016). Esta situación también coincide con lo mencionado por Heads (2004), donde una característica de los NP es que pueden tener alta diversidad. Sin embargo, aún es necesario establecer la existencia de correlaciones entre los sesgos y los patrones identificados.

En la caracterización ambiental, los NPP se distribuyeron equitativamente en dos tipos de rocas (ígneas y sedimentarias; nueve NP por cada tipo de roca), lo cual coincide parcialmente con los hallazgos de Escalante et al. (2004). En particular, los Altos de Chiapas y la Sierra Juárez poseen la mayor diversidad de rocas en comparación con otros sitios de México, por lo que dan un indicio acerca de su complejidad geológica. Respecto a la topografía, los NPP se localizaron en intervalos altitudinales que van desde los 200 a los 4,000 m, siendo el intervalo de 1,500 a 2,500 m donde se presentó la mayor cantidad de NPP, coincidiendo con los hallazgos de Escalante et al. (2004) y Gámez et al. (2012). Los Altos de Chiapas y la Sierra Juárez tuvieron el mayor número de contrastes altitudinales (seis y cuatro, respectivamente) en comparación con otros sitios, lo que indica mayor complejidad orográfica. Sin embargo, hay que destacar que los nodos fusionados fueron los que 
ocuparon las altitudes más bajas, lo cual podría indicar un problema metodológico durante el proceso de fusión.

Respecto al clima, los Altos de Chiapas y la Sierra Juárez presentaron los mayores contrastes. La mayoría de los NPP se distribuyeron en climas templados, lo cual coincide con el trabajo de Escalante et al. (2004). En cuanto a los tipos de suelo, los NPP también mostraron variabilidad, siendo los Altos de Chiapas en donde se puede inferir que las geoformas parecen tener una topografía compleja, misma que se refleja en la variabilidad de los suelos. La mayoría de los NP se ubicaron sobre acrisoles, los cuales son un grupo de suelos que presenta mayor contenido de arcilla en el subsuelo que en el suelo superficial (FAO, 2007). En México, estos suelos se usan en la agricultura con rendimientos muy bajos, salvo algunos árboles frutales (cuyos rendimientos van de medios a altos); pero también se usan en la ganadería con pastos inducidos (INEGI, 2017c).

La complejidad de los NP también pudo observarse mediante las cuencas hidrográficas. En particular, la gran cantidad de NPP de los Altos de Chiapas y en Cuicatlán-Sierra Juárez corresponde a los ríos más caudalosos del país (Conagua-Semarnat, 2008; Pérez et al., 2010), indicando gran complejidad hidrográfica. Con relación a la vegetación, la mayoría de los NPP se ubicó en zonas boscosas, coincidiendo con García-Marmolejo et al. (2008), siendo los Altos de Chiapas y la Sierra Juárez las áreas geográficas que presentaron los mayores contrastes entre tipos de vegetación y uso de suelo, por lo que en conjunto esas áreas presentan gran complejidad en sus condiciones ambientales. Cabe mencionar que, en términos de conservación, lamentablemente muchos de los NPP están ubicados en o cerca de áreas cuya vegetación natural ha sido removida.

La caracterización de los NP brinda información útil para robustecer la importancia de los NPP reconocidos en esta investigación, que pueden incorporarse a estrategias de conservación. Más del $25 \%$ de la superficie de las áreas protegidas del país incluyen territorios indígenas, por lo que la conservación de estos espacios depende de las poblaciones nativas, las cuales debe ser involucradas en los planes de manejo (Sarukhán et al., 2009). Existen diversas prácticas y mecanismos ecológico-sociales en el conocimiento tradicional de las comunidades indígenas, que si bien, su objetivo no es la conservación, si es una consecuencia (Berkes et al., 2000). Por lo tanto, la caracterización cultural mediante la identificación de los pueblos indígenas que habitan en los NP, es indispensable para garantizar su conservación. Un problema primordial al realizar la caracterización cultural es que no existía información digital sobre la dimensión espacial de los pueblos indígenas. Algunos documentos como el de Boege (2008) y el de la Comisión Nacional para el Desarrollo de los Pueblos Indigenas (CDI, 2006), sólo muestran de manera general sus áreas de distribución, sin especificar todos los municipios o localidades que los pueblos habitan, lo que genera ciertas limitaciones. La información almacenada en el Atlas Nacional de Pueblos Indígenas (CDI, 2017) sólo menciona los municipios o regiones más importantes, y muchas veces las referencias geográficas no son del todo claras. Así mismo es importante señalar que los pueblos indígenas no ocupan la totalidad de esas áreas, lo cual corresponde con el documento de la CDI (2006). La distribución discontinua de los pueblos indígenas puede deberse a aspectos sociales como: volumen de población indígena, patrones de asentamiento, migración, entre otras 
características; por lo que es importante tenerlos en cuenta al intentar cartografiar sus áreas de distribución (Comas, 1980; CDI, 2006). Recientemente, en el Atlas Nacional de los Pueblos Indígenas (CDI, 2020) se ha integrado información digital que permite descargar la distribución geográfica de los pueblos indígenas, con las cuales se puede valorar la incertidumbre de las áreas de distribución de dichos pueblos presentadas en esta investigación. La cartografía realizada en este trabajo tiene como ventaja el contribuir en la obtención de información digital acerca de las áreas de distribución de los pueblos indígenas de México, siendo esta investigación pionera en este tipo de estudios. En el Atlas Nacional de los Pueblos Indígenas (CDI, 2020) existe información digital reciente acerca de la distribución de estos pueblos, sin embargo dicha distribución es de tipo punto y no se especifica el área que ocupan, aspecto que sí abordamos en nuestro mapa. Por otro lado, es importante mencionar que nuestra cartografía sólo refleja algunos municipios mencionados en el Atlas Nacional de los Pueblos Indígenas (CDI, 2017); por lo que se requiere corroborar la presencia de los pueblos indígenas en la totalidad de cada municipio, siendo necesario realizar estudios detallados acerca de este tema.

Con respecto a los NPP, se identificaron cuatro grupos indígenas en el estado de Oaxaca, dos de ellos se superponen en Cuicatlán y la Sierra Juárez, generando una mezcla de pueblos que ocupan un mismo espacio, por lo que pueden considerarse como zonas complejas desde un punto de vista antropológico-cultural, siendo el grupo Zapoteco donde se distribuye la mayoría de los NPP (Fig. 9). En los Altos de Chiapas se reconocieron dos pueblos, al igual que en el Soconusco; la mayoría de los NPP se ubicaron en los pueblos de los Mames y los Mochos (Soconusco) donde la distribución de este último queda anidada dentro de la distribución de los Mames, lo que genera cierta complejidad cultural.

El estado de Oaxaca es el que presenta mayor complejidad geológica y geomorfológica (Hernández et al., 2009), así mismo es el estado que presenta mayor población indígena (Boege, 2008), seguido por el estado de Chiapas (Conabio, 2012). Ambos estados tienen la mayor diversidad biológica del país (Arita et al., 1997; Boege, 2008) y poseen las áreas geográficas (político-administrativas) con mayor número de NPP, lo cual indica gran complejidad natural y cultural. No obstante, dicha complejidad natural-cultural no implica necesariamente que los habitantes indígenas tengan buena calidad de vida; ya que lamentablemente los dos estados antes mencionados son los más pobres a nivel nacional (Conabio, 2010).

Respecto a los mamíferos incluidos en los NPP, de las 30 especies, una es un conejo, tres son murciélagos, cuatro musarañas y 22 son roedores (incluyendo tres ardillas). A pesar de que nosotros no identificamos una población indígena coincidente con el conejo Sylvilagus cunicularius, esta especie es ampliamente cazada en México para aprovecharla como piel, alimento y medicina (Gilcrease, 2014). Otra especie aprovechada como alimento y artesanía es la ardilla gris mexicana Sciurus aureogaster (García et al., 2014), la cual coincidió en NPP con pueblos como los Zapotecos, Choles, Mames y Mochos. Finalmente, al roedor Sigmodon mascotensis en ocasiones se le considera una plaga que puede causar daños menores a los cultivos (Schnell et al., 2010). Esta especie se ubica en NPP que coinciden con Zapotecos. En general, la información sobre el uso y aprovechamiento de las especies de los NP con los pueblos indígenas es escasa, ya que la 
mayoría son especies poco conspicuas y utilizadas, además que no hay suficientes estudios al respecto.

De las 30 especies de los NPP, ocho se encuentran categorizadas en riesgo en la Norma Oficial Mexicana 059 (NOM-059; Semarnat, 2010; DOF, 2014): cinco en la categoría Sujeta a Protección Especial (Cryptotis alticola, Handleyomys chapmani, Heteromys nelsoni, Peromyscus zarhynchus y Tylomys tumbalensis) y tres en la categoría Amenazada (Megadontomys cryophilus, Pteronotus gymnonotus y Sorex veraepacis). En general, muchas especies de mamíferos no han sido apropiadamente evaluadas, ya que solo el 10\% de la NOM-059 tienen soporte técnico que justifica su inclusión y categorización en la Norma (García-Aguilar et al., 2017). Por ejemplo, Sylvilagus cunicularius, a pesar de estar restringida a territorios altamente específicos, mostrar amplia pérdida de hábitat y estar sujeta a cacería, no ha sido categorizada como en riesgo (Velázquez, 2012; Gilcrease, 2014).

En este trabajo consideramos que el Análisis de Trazos, la priorización y la caracterización físico-geográfica y cultural de los NP pueden ser útiles para contribuir tanto en la conservación de los patrones y procesos biogeográficos que generan la biodiversidad, así como en preservar la mayor cantidad de recursos naturales y culturales. Los siguientes pasos en la conservación de estas áreas prioritarias requerirán realizar trabajo de campo para verificar las condiciones ambientales y la congruencia en la presencia de los mamíferos y los pueblos indígenas en los NPP. Al realizar el trabajo de campo también se podrían visitar las poblaciones indígenas para llevar a cabo entrevistas y documentar el conocimiento sobre las especies $y$, con base en ello, realizar interpretaciones acerca de las formas de la apropiación de la mastofauna, la cual puede brindar información para preservar tanto la biodiversidad, como otros recursos naturales implicados. De esta manera, se pretende lograr en el futuro un manejo adecuado de áreas geográficas complejas como Los Altos de Chiapas y la Sierra Juárez. Esta última se considera como el ecosistema más extenso y conservado de México, además de presentar una alta biodiversidad (HernándezRodríguez et al., 2019; Pacheco-Cruz et al., 2019). Cuicatlán se caracteriza por tener una accidentada orografía que no permite la libre movilidad de personas y donde se practican actividades agrícolas (Ramos et al., 2012). Por otro lado, el NPP de la zona meridional de la Sierra Madre del Sur es reconocida como una zona ideal para producir agave y mezcal por sus características agroclimáticas (Bautista \& Ramírez, 2005). Por último, el Soconusco es un área económica importante, pero presenta la más alta peligrosidad de riesgo sísmico en México, así como un alto grado de exposición a fenómenos hidrometeorológicos (Chiapas, 2020).

La crisis y las tasas de extinción que actualmente presenta la biodiversidad son de las problemáticas más urgentes que enfrenta la humanidad. Si esta tendencia continúa, los patrones de distribución geográfica de las especies en el futuro serán modificados y, en consecuencia, las estrategias de conservación mediante la priorización de los NP serán ineficientes. La posibilidad de involucrar los aspectos sociales, culturales y de la biodiversidad, pueden tener como punto de partida estos sitios con alta correlación de las diferentes diversidades. 
AgRADECIMIENTOS. Investigación realizada gracias al Programa UNAM-DGAPA-PAPIIT Proyecto IN217717. Agradecemos a los revisores anónimos y al editor por sus sugerencias para mejorar el manuscrito.

\section{LITERATURA CITADA}

Aguilar-Aguilar, R., Contreras, R. (2001) La distribución de los mamíferos marinos de México: Un enfoque panbiogeográfico. Pp. 213-2219. En: J. Llorente-Bousquets, J. J. Morrone (Eds.). Introducción a la Biogeografía en Latinoamérica: Teorías, conceptos, métodos y aplicaciones. Las Prensas de Ciencias, UNAM.

Almendra, A. L., Rogers, D. S., González-Cózatl, F. X. (2014) Molecular phylogenetics of the Handleyomys chapmani complex in Mesoamerica. Journal of Mammalogy, 95 (1), 26-40. https://doi.org/10.1644/13-MAMM-A-044.1

Álvarez-Mondragón, E., Morrone, J. J. (2004) Propuesta de áreas para la conservación de aves de México, empleando herramientas panbiogeográficas e índices de complementariedad. Interciencia, 29, 112-120.

Arita, H., Figueroa, F., Frisch, A., Rodríguez, P., Santos-Del-Prado, K. (1997) Geographical range size and the conservation of Mexican mammals. Conservation biology, 11, 92-100. https://doi.org/10.1046/j.1523-1739.1997.95274.x

Barrasa, G. S. (2013) Conocimientos y usos tradicionales de la fauna en dos comunidades campesinas de la Reserva de la Biosfera Encrucijada. Etnobiología, 11, 16-28.

Bautista, J. A., Ramírez, J. (2005) Sostenibilidad y pobreza en las unidades socioeconómicas campesinas de la "región del mezcal" en Oaxaca. Pp. 325-351. En: R. R. Wences, R. L. Sampedro, U. R. López, A. J. L. Rosas (Eds.). Problemática territorial y ambiental en el desarrollo regional. Universidad Michoacana de San Nicolás de Hidalgo, Morelia.

Berkes, F., Colding, J., Folke, C. (2000) Rediscovery of traditional ecological knowledge as adaptive management. Ecological Applications, 10, 1251-1261.

Boege, E. (2008) El patrimonio biocultural de pueblos indígenas de México: Hacia la conservación in situ de la biodiversidad y agrodiversidad en los territorios indígenas. Instituto Nacional de Antropología e Historia, Comisión Nacional para el Desarrollo de los Pueblos Indígenas. México, $344 \mathrm{pp}$.

Bolkovic, M. L. (1997) Usos de fauna silvestre de pobladores de las cercanías de la Reserva Provincial Copo, Santiago del Estero, Argentina. En: T. G. Fang, R. E. Bodmer, R. Aquino, M. $\mathrm{H}$. Valqui (Eds.). Manejo de la fauna silvestre en la Amazonia. Siglo XXI, México D.F.

CDI (2006) Regiones indígenas de México. México. Disponible en: www.cdi.go.mx (Consultado: 15 noviembre 2017).

CDI (2017) Atlas de los Pueblos Indígenas de México. México. Disponible en: http://www.cdi.gob.mx/atlas/ (Consultado: 15 noviembre 2017).

CDI (2018) Atlas de los Pueblos Indígenas de México, Pueblos Indígenas (Galería de Imágenes). México. Disponible en: http://www.cdi.gob.mx/atlas2015/?page_id=67 (Consultado: 14 junio 2018).

CDI (2020) Atlas Nacional de los Pueblos Indígenas. México. Disponible en: https://www.youtube.com/watch?v=3K0i2pb51MY (Consultado: 20 mayo 2021). 
Chiapas, H. (2020) Programa Regional de Desarrollo. Región X Soconusco. México. Disponible en: http://www.haciendachiapas.gob.mx/planeacion/Informacion/Desarrollo-Regional/progregionales/SOCONUSCO.pdf (Consultado: 20 septiembre 2020).

Colwell, R. K., Coddington, J. A. (1994) Estimating terrestrial biodiversity through extrapolation. Philosophical Transactions of the Royal Society London B, 345, 110-118. https://doi.org/10.1098/rstb.1994.0091

Comas, J. (1980) Origen de las culturas precolombinas. Secretaría de Educación Pública, México, $158 \mathrm{pp}$.

CONABIO (2010) Grados de marginación por entidad federativa. Disponible en: http://www.conabio.gob.mx/informacion/gis/ (Consultado: 1 diciembre 2017).

CONABIO (2012) Población indígena de México por Entidad Federativa. Disponible en: http://www.conabio.gob.mx/informacion/gis/ (Consultado: 1 diciembre 2017).

CONABIO (2017) Portal de Geoinformación: Sistema Nacional de información sobre la Biodiversidad. Disponible en: http://www.conabio.gob.mx/informacion/gis/ (Consultado: 15 diciembre 2017).

CONAGUA-SEMARNAT (2008) Informe de la situación del medio ambiente en México. Compendio de estadística ambiental. Ciudad de México, México.

Contreras, R., Morrone, J. J., Luna-Vega, I. (2001) Biogeographic methods identify gymnosperm biodiversity hotspots. Naturwissenschaften, 88, 427-430.

http://dx.doi.org/10.1007/s001140100252

Corona, A. M., Morrone, J. J. (2005) Track analysis of the species of Lampetis (Spinthoptera) Casey, 1909 (Coleoptera: Buprestidae) in North America, Central America, and the West Indies. Caribbean Journal of Science, 41, 37-41.

http://dx.doi.org/10.1111/j.1365-2699.2009.02126.x

Corona, A. M., Toledo, V. H. (2006) Patrones de distribución de la familia Buprestidae (Coleoptera). Pp. 333-391. En: J. J. Morrone, J. Llorente-Bousquets (Eds.). Componentes bióticos principales de la entomofauna mexicana. Las Prensas de Ciencias, UNAM, México, México.

Craw, R. C., Grehan, J. R., Heads, M. J. (1999) Panbiogeography: tracking the history of life. University Press, Oxford, 299 pp.

Croizat, L. (1958) Panbiogeography. Vol. 1 y 2. Caracas: Publicado por el autor.

DOF (2014) Acuerdo por el que se da a conocer la lista de especies y poblaciones prioritarias para la conservación. Estados Unidos Mexicanos. Secretaría de Medio Ambiente y Recursos Naturales. Ciudad de México, México.

EROS (2010) 30 arc-second DEM of North America. Disponible en: https://databasin.org/datasets/d2198be9d2264de19cb93fe6a380b69c (Consultado: 4 marzo 2017).

Escalante, T. (2001) Avances en el atlas biogeográfico de los mamíferos terrestres de México. Pp. 297-302. En: J. Llorente-Bousquets, J. J. Morrone (Eds.). Introducción a la Biogeografía en Latinoamérica: Teorías, Conceptos, Métodos y Aplicaciones. Las prensas de Ciencias, UNAM, México.

Escalante, T., Morales, R. (2015) Biogeografía de la Conservación: Prioridades y Desafíos. Biogeografía, 8, 36-44. 
Escalante, T., Noguera-Urbano, E. A., Corona, W. (2018) Track analysis of the Nearctic region: Identifying complex areas with mammals. Zoological Systematics and evolutionary research, $1-12$. http://dx.doi.org/10.1111/jzs.12211

Escalante, T., Noguera-Urbano, E. A., Pimentel, B., Aguado-Bautista, O. (2017) Methodological issues in modern track analysis. Evolutionary Biology, 44, 284-293. http://dx.doi.org/10.1007/s11692-016-9401-8

Escalante, T., Rodríguez-Tapaia, G., Morrone, J. J. (2004) The diversification of Nearctic mammals in the Mexican Transition Zone. Biological Journal of the Linnean Society, 83, 327339.

http://dx.doi.org/10.1111/j.1095-8312.2004.00386.x

ESRI (2012) ArcGIS 10.1. Disponible en: http://resources.arcgis.com/es/help/gettingstarted/articles/026n00000012000000.htm (Consultado: 03 abril 2017).

ESRI (2020) ArcMap. Disponible en: https://desktop.arcgis.com/es/arcmap/ (Consultado: 31 mayo 2020).

Estrada-Portillo, D., Rosas-Rosas, O. C., Parra-Inzunza, F., Guerrero-Rodríguez, J. D., Tarango-Arámbula, L. A. (2018) Valor de uso, importancia cultural y percepciones sobre mamíferos silvestres medianos y grandes en la mixteca poblana. Acta Zoológica Mexicana (nueva serie), 34, 1-15.

http://dx.doi.org/10.21829/azm.2018.3412131

FAO (2007) Base Referencial Mundial del Recurso Suelo, Informes sobre Recursos Mundiales de Suelos. $117 \quad$ pp. Disponible en: http://scholar.google.com/scholar?hl=en\&btnG=Search\&q=intitle:Base+referencial+mun dial+del+recurso+suelo\#0 (Consultado: 04 abril 2020).

Fortino, A., Morrone, J. J. (1997) Signos gráficos para la representación de análisis panbiogeográficos. Biogeographica, 73, 49-56.

Gámez, N., Escalante, T., Rodríguez, G., Linaje, M., Morrone, J. J. (2012) Caracterización biogeográfica de la Faja Volcánica Transmexicana y análisis de los patrones de distribución de su mastofauna. Revista Mexicana de Biodiversidad, 83, 258-272. http://dx.doi.org/10.22201/ib.20078706e.2012.1.786

García, E., CONABIO (1998) Climas. Disponible en: http://www.conabio.gob.mx/informacion/metadata/gis/clima1mgw.xml?_httpcache=yes\& _xsl=/db/metadata/xsl/fgdc_html.xsl\&_indent=no (Consultado: 10 noviembre 2017).

García, F. A. (2008) La etnozoología como una alternativa para el desarrollo comunitario sustentable en la Reserva Estatal Sierra de Montenegro, Morelos, México. Facultad de Ciencias Agropecuarias. Universidad Autónoma del Estado de Morelos, Cuernavaca, Morelos, México.

García, A., Lozano, M. A., Ortiz, A. L., Monroy, R. (2014) Uso de mamíferos silvestres por habitantes del Parque Nacional El Tepozteco, Morelos, México. Etnobiología, 12, 57-67.

García-Aguilar, M. C., Luévano-Esparza, J., De la Cueva, H. (2017) La fauna nativa de México en riesgo y la NOM-059: ¿Están todos los que son y son todos los que están? Acta Zoológica Mexicana (nueva serie), 33, 188-198.

http://dx.doi.org/10.21829/azm.2017.3321060 
García-Barros, E., Guerra, P., Luxiáñez, M., J Cano, J. M., Munguía, M. L., Moreno, J. C., Sainz, H., Sanz, M. J., Simón, C. J. (2002) Parsimony analysis of endemicity and its application to animal and plant geographical distributions in the Ibero-Balearic region (western Mediterranean). Journal of Biogeography, 29, 109-124.

https://doi.org/10.1046/j.1365-2699.2002.00653.x

García-Marmolejo, G., Escalante, T., Morrone, J. J. (2008) Establecimiento de prioridades para la conservación de mamíferos terrestres neotropicales de México. Mastozoología Neotropical, 15, 41-65.

http://dx.doi.org/0327-9383

Gilcrease, K. (2014) The Mexican cottontail (Sylvilagus cunicularius): A historical perspective of hunting and grazing and implications for conservation planning. Acta Zoológica Mexicana (nueva serie), 30, 32-40.

http://dx.doi.org/10.21829/azm.2014.301127

González-Ávila, A., Contreras-Medina, R., Espinosa-Organista, D., Luna-Vega, I. (2017) Track analysis of the order gomphales (Fungi: Basidiomycota) in Mexico. Phytotaxa, 316, 22-38. http://dx.doi.org/10.11646/phytotaxa.316.1.2

González, J. A., Vallejo, J. R. (2014) Vertebrados silvestres usados en la medicina popular del sector centro-occidente de España: una revisión bibliográfica. Etnobiología, 12, 1-22.

Grehan, J. R. (1989) Panbiogeography and conservation science in New Zealand. Journal of Zoology, 16, 731-748.

http://dx.doi.org/10.1080/03014223.1989.10422930

Grehan, J. R. (1993) Conservation biogeography and the biodiversity crisis: a global problem in space/time. Biodiversity Letters, 1, 134-140.

http://dx.doi.org/10.2307/2999686

Heads, M. J. (1998) Integrating earth and life sciences in New Zealand natural history: The parallel arcs model. Journal of Zoology, 16, 549-585.

http://dx.doi.org/10.1080/03014223.1989.10422922

Heads, M. J. (2004) What is a node? Journal of Biogeography, 31, 1883-1891. http://dx.doi.org/10.1111/j.1365-2699.2004.01201.x

Hernández, J., Ortiz, M. A., Figueroa, M. (2009) Análisis morfoestructural del estado de Oaxaca, México: un enfoque de clasificación tipológica del relieve. Investigaciones Geográficas, Boletín del Instituto de Geografía, 68, 7-24.

http://dx.doi.org/10.14350/rig.17995

Hernández-Rodríguez, E., Escalera-Vázquez, L., Calderón-Patrón, J. M., Mendoza, E. (2019) Mamíferos medianos y grandes en sitios de tala de impacto reducido y de conservación en la Sierra Juárez, Oaxaca. Revista Mexicana de Biodiversidad, 90, 1-10.

http://dx.doi.org/10.22201/ib.20078706e.2019.90.2776

Hortal, J., De Bello, F., Diniz-Filho, J. A. E., Lewinsohn, T. M., Lobo, J. M., Ladle, R. L. (2015) Seven shortfalls that beset large-scale knowledge of biodiversity. Annual Review of Ecology, Evolution, and Systematics, 46, 523-549.

http://dx.doi.org/10.1146/annurev-ecolsys-112414-054400

Huber, O., Riina, R. (2003) Glosario fitoecológico de las Américas: México, América Central e islas del caribe: Países hispanohablantes. UNESCO-CoroLab Humbolt. París, 474 pp. 
Hull, D. L. (1988) Science as a process: An evolutionary account of the social and conceptual development of science. University of Chicago Press. Chicago.

INEGI (2000) Acala. Disponible http://www.beta.inegi.org.mx/app/biblioteca/ficha.html?upc=702825657345 (Consultado: 9 septiembre 2017).

INEGI (2001a) Llano Grande. Disponible en: http://www.beta.inegi.org.mx/app/biblioteca/ficha.html?upc=702825666507 (Consultado: 11 septiembre 2017).

INEGI (2001b) Motozintla de Mendoza. Disponible en: http://www.beta.inegi.org.mx/app/biblioteca/ficha.html?upc=702825700027 (Consultado: 9 septiembre 2017).

INEGI (2001c) San Cristóbal de las Casas. Disponible en: http://www.beta.inegi.org.mx/app/biblioteca/ficha.html?upc=702825657352 (Consultado: 9 septiembre 2017).

INEGI (2001d) San Juan Quiotepec. Disponible en: http://www.beta.inegi.org.mx/app/biblioteca/ficha.html?upc=702825666163 (Consultado: 9 septiembre 2017).

INEGI (2002) Escuintla. Disponible http://www.beta.inegi.org.mx/app/biblioteca/ficha.html?upc=702825701833 (Consultado: 9 septiembre 2017).

INEGI (2003) San Agustín Loxicha. Disponible en: http://www.beta.inegi.org.mx/app/biblioteca/ficha.html?upc=702825705442 (Consultado: 11 septiembre 2017).

INEGI (2004a) Ixhuatán. Disponible http://www.beta.inegi.org.mx/app/biblioteca/ficha.html?upc=702825708504 (Consultado: 9 septiembre 2017).

INEGI (2004b) Jitotol. Disponible en: http://www.beta.inegi.org.mx/app/biblioteca/ficha.html?upc=702825708542 (Consultado: 9 septiembre 2017).

INEGI (2004c) Tumbalá. Disponible http://www.beta.inegi.org.mx/app/biblioteca/ficha.html?upc=702825708054 (Consultado: 9 septiembre 2017).

INEGI (2014a) Ciudad Guzmán. Disponible http://www.beta.inegi.org.mx/app/biblioteca/ficha.html?upc=702825205973 (Consultado: 10 septiembre 2017).

INEGI (2014b) Conjunto de datos vectoriales edafológico. Disponible en: http://www.conabio.gob.mx/informacion/gis/ (Consultado: 10 noviembre 2017).

INEGI (2014c) Cuicatlán. Disponible en: http://www.beta.inegi.org.mx/app/biblioteca/ficha.html?upc=702825206796 (Consultado: 9 septiembre 2017).

INEGI (2014d) Guía para la interpretación de cartografía de uso del suelo y vegetación. Disponible en:

http://internet.contenidos.inegi.org.mx/contenidos/Productos/prod_serv/contenidos/esp 
anol/bvinegi/productos/nueva_estruc/702825092030.pdf (Consultado: 9 septiembre 2017).

INEGI (2016a) División política estatal, 1:250000. Disponible en: http://www.conabio.gob.mx/informacion/gis/ (Consultado: 16 noviembre 2017).

INEGI (2016b) División política municipal, 1:250000. Disponible en: http://www.conabio.gob.mx/informacion/gis/ (Consultado: 16 noviembre 2017)

INEGI (2017a) Mapa Virtual de México. Disponible en: http://gaia.inegi.org.mx/mdm6/ (Consultado: 10 noviembre 2017).

INEGI (2017b) Datos vectoriales. Rocas. Disponible en: http://www.inegi.org.mx/geo/contenidos/recnat/geologia/infoescala.aspx (Consultado: 9 septiembre 2017).

INEGI (2017c) Unidades y subunidades de suelo. Guía para la Interpretación de Cartografía Edafología. Disponible en: http://www.inegi.org.mx/inegi/SPC/doc/INTERNET/Edaflll.pdf (Consultado: 9 noviembre 2017).

INEGI (2018) Continuo de Elevaciones Mexicano (CEM). Disponible en: http://www.beta.inegi.org.mx/app/geo2/elevacionesmex/ (Consultado: 8 diciembre 2018).

INEGI, Lugo-Hupb, J., Vidal-Zepeda, R., Fernández-Equiarte, A., Gallegos-García, A., ZavalaH., J. (1990) Hipsometría. Disponible en: http://www.conabio.gob.mx/informacion/gis/ (Consultado: 10 noviembre 2017).

INEGI, INE, Conagua, Priego, A. G., Isunza, E., Luna, N., Pérez, J. L. (2007) Cuencas Hidrográficas de México. Disponible en: http://www.conabio.gob.mx/informacion/gis/

Lorenzo, M. C., Cruz, L., Naranjo, L. E. J., Barragán, F. (2007) Uso y conservación de mamíferos silvestres en una comunidad de las cañadas de la Selva Lacandona, Chiapas, México. Etnobiología, 5, 99-107.

Luna-Vega, I., Morrone, J. J., Escalante, T. (2010) Conservation biogeography: A viewpoint from evolutionary biogeography. Pp. 229-240. En: M. Gailis, S. Kalninjs (Eds.). Biogeography. Nova-Science Publishers, Nueva York.

MacDonald, G. M. (2003) Biogeography: Introduction to Space, Time, and Life. John Wiley \& Sons. Nueva York, 528 pp.

Maderey, L., Torres-Ruata, C. (1990) Hidrografía. Disponible en: http://www.conabio.gob.mx/informacion/gis/ (Consultado: 10 noviembre 2017).

Márquez, J., Morrone, J. J. (2003) Análisis panbiogeográfico de las especies de Heterolinus y Homalolinus (Coleoptera: Staphylinidae: Xantholinini). Acta Zoológica Mexicana (nueva serie), 90, 15-25.

http://dx.doi.org/10.1002/ejoc.200800178

Miguel-Talonia, C., Escalante, T. (2013) Los nodos: El aporte de la panbiogeografía al entendimiento de la biodiversidad. Biogeografía, 6, 30-42.

Monroy, R., García-Flores, A. (2013) La fauna silvestre con valor de uso en los huertos frutícolas tradicionales de la comunidad indígena de Xoxocotla, Morelos, México. Etnobiología, 11, 44-52.

Morales, G. P. (2000) Cacería de subsistencia en tres comunidades de la zona maya de México y Guatemala. El Colegio de la Frontera Sur (ECOSUR). Quintana Roo, México, 52 pp. 
Morrone, J. J. (1999) How can biogeography and cladistics interact for the selection of areas for biodiversity conservation?: A view from Andean weevils (Coleoptera: Curculionidae). Biogeographica, 75, 89-96.

Morrone, J. J. (2000) La importancia de los atlas biogeográficos para la conservación de la biodiversidad. Pp. 69-78. En: F. Martín-Piera, J. J. Morrone, A. Melic (Eds.). Hacia un Proyecto CYTED para el Inventario y Estimación de la Diversidad Entomológica en Iberoamérica. Physis (Buenos Aires), México.

Morrone, J. J. (2001) Sistemática, biogeografía y evolución. Los patrones de la biodiversidad en tiempo-espacio. Museo de Zoología "Alfonso L. Herrera", UNAM, Ciudad de México, México.

Morrone, J. J. (2015) Track analysis beyond panbiogeography. Journal of Biogeography, 42, 413425.

http://dx.doi.org/10.1111/jbi.12467

Morrone, J. J. (2019) Regionalización biogeográfica y evolución biótica de México: Encrucijada de la biodiversidad del Nuevo Mundo. Revista Mexicana de Biodiversidad, 90, 1-68. http://dx.doi.org/10.22201/ib.20078706e.2019.90.2980

Morrone, J. J., Escalante, T. (2016) Introducción a la biogeografía. Las Prensas de Ciencias, UNAM, México, 315 pp.

Morrone, J. J., Escalante, T., Rodríguez-Tapia, G. (2017) Mexican biogeographic provinces: Map and shapefiles. Zootaxa, 4277, 277-279.

http://dx.doi.org/10.11646/zootaxa.4277.2.8

Morrone, J. J., Espinosa-Organista, D. (1998) La relevancia de los atlas biogeográficos para la conservación de la biodiversidad mexicana. Ciencia, 49, 12-16.

Morrone, J. J., Márquez, J. (2003) Aproximación a un atlas biogeográfico mexicano: Componentes bióticos principales y provincias biogeográficas. Pp. 217-220. En: J. LlorenteBousquets, J. J. Morrone (Eds.). Introducción a la Biogeografía en Latinoamérica: Teorías, Conceptos, Métodos y Aplicaciones. Las prensas de Ciencias UNAM, México.

Natural Earth (2017a) Land polygons inlcuding major islands, 1:10 000. Disponible en: http://www.naturalearthdata.com/downloads/10m-physical-vectors/10m-land/ (Consultado: 1 enero 2017).

Natural Earth (2017b) Natural and artificial lakes, 1:50 000. Disponible en: http://www.naturalearthdata.com/downloads/50m-physical-vectors/ (Consultado: 4 marzo 2017).

Natural Earth (2017c) Ocean polygon split into contiguous pieces, 1:50 000. Disponible en: http://www.naturalearthdata.com/downloads/50m-physical-vectors/50m-ocean/ (Consultado: 4 marzo 2017).

Ochoa, L., Cruz, B., García-Marmolejo, G., Luis-Martínez, A. (2003) Contribución al atlas panbiogeográfico de México: los géneros Adelpha y Hamadryas (Nymphalidae), y Dismorphia, Enantia, Lienix y Pseudopieris (Pieridae) (Papilionoidea; Lepidoptera). Folia Entomológica Mexicana, 42, 65-77.

Oliveira, U., Paglia, A. P., Brescovit, A. D., de Carvalh, C. J. B., Silva, D. P., Rezende, D. T., Leite, F. S. F., Batista, J. A. N., Barbosa, J. P. P. P., Stehmann, J. R., Ascher, J. S., de Vasconcelos, M. F., De Marco, P, Jr., Löwenberg-Neto, P., Dias, P. G., Ferro, V. G., 
Santos, A. J. (2016) The strong influence of collection bias on biodiversity knowledge shortfalls of Brazilian terrestrial biodiversity. Diversity and Distributions, 22, 1232-1244. http://dx.doi.org/10.1111/ddi.12489

Pacheco-Cruz, N., Clark-Tapia, R., Campos, J. E., Gorgonio-Ramírez, M., Luna-Krauletz, M. D., Naranjo-Luna, F., Corrales, A., Alfonso-Corrado, C. (2019) Regeneration of Oreomunnea mexicana (Juglandaceae), a threatened relict species of the Sierra Juarez cloud forest, Oaxaca, Mexico. Madera y Bosques, 25, 1-14.

http://dx.doi.org/10.21829/myb.2019.2531852

Pérez, A. G., Cuevas, M. L, Cotler, H., González, D. I., Tharme, R. (2010) Evaluación del grado de alteración ecohidrológica de los ríos y corrientes superficiales de México. Investigación ambiental, 2, 25-46.

Pérez, B., Cruz, Á., Batalla, J. J. (2008) América Precolombina: El despertar de los testigos mudos. Edimat, Madrid, 298 pp.

Pérez, G. R. (1998) Una contribución para la comprensión de los usos, valores y tipos de importancia que representan los vertebrados terrestres de México. En: D. H. Benítez, E. V. López, P. A. Jiménez, S. A. (Eds.). Aspectos económicos sobre la biodiversidad de México. Comisión Nacional Para el Conocimiento y Uso de la Biodiversidad (CONABIO).

Pérez, G. R., Jaramillo, F., Muñiz, A., Torres, M. (1995) Importancia económica de los vertebrados silvestres de México. Comisión Nacional Para el Conocimiento y Uso de la Biodiversidad, $170 \mathrm{pp}$.

QGIS Development Team (2016) QGIS Geographic Information System. Disponible en: http://qgis.org/es/site/ (Consultado: 27 febrero 2017).

Ramírez Pulido, J., Arroyo-Cabrales, J., Gardner, A. L., González-Ruiz, N. (2014) List of recent land mammals of Mexico. Tech University Natural Science Research Laboratory. Texas, 68 pp.

http://dx.doi.org/10.5962/bhl.title.142891

Ramírez-Pulido, J., González-Ruiz, N., Ameneyro, G., Castro-Campillo, A., Salame-Méndez, A. (2016) Panorama del conocimiento de los mamíferos de México: con énfasis a nivel estatal. Pp. 39-62. En: M. Y. Briones-Salas, G. Hortelano-Moncada, G. S. R. Magaña-Cota, G. Sánchez-Rojas, J. E. Sosa-Escalante (Eds.). Riqueza y conservación de los mamíferos en México a nivel estatal. Instituto de Biología, Universidad Nacional Autónoma de México, Asociación Mexicana de Mastozoología, A.C. y Universidad de Guanajuato, Ciudad de México.

Ramos, A. L., Velásquez, R. M., Sánchez, M. A., Hernández, A. (2012) Tipos de regiones en el distrito de Cuicatlán del estado de Oaxaca. Contribuciones a las Ciencias Sociales.

Rojas-Parra, C. A. (2007) Una herramienta automatizada para realizar análisis panbiogeográficos. Biogeografía, 1, 31-33.

Rojas, A. E., Moreno, C. E. (2014) Los servicios ambientales que generan los mamíferos silvestres. Universidad Autónoma del Estado de Hidalgo. Boletín Científico de Ciencias Básicas e Ingeniería, No. 3. Disponible en: http://www.uaeh.edu.mx/scige/boletin/icbi/n3/e10.html (Consultado: 08 marzo 2016).

Rzedowski, J. (1978) Vegetación de México. México: Limusa. 
Sánchez, O. (2011) La importancia de las escalas de espacio y de tiempo en la conservación de vida silvestre. Pp. 13-47. En: O. Sánchez, P. Zamorano, E. Peters, H. Moya (Eds.). Temas sobre conservación de vertebrados silvestres en México. INE-SEMARNAT. Ciudad de México.

Sarukhán, J., Koleff, P., Carabias, J., Soberón, J., Dirzo, R., Llorente-Bousquets, J., Halffter, G., González, R., March, I., Mohar, S., Anta, J., De la Maza, J. (2009) Capital Natural de México. Síntesis: conocimiento actual, evaluación y perspectivas de sustentabilidad. Comisión Nacional para el Conocimiento y Uso de la Biodiversidad, México, D.F.

Schnell, G. D., De Lourdes Romero-Almaraz, M., Martínez-Chapital, S. T., SánchezHernández, C., Kennedy, M. L., Best, T. L., Wooten, M. C., Owen, R. D. (2010) Habitat use and demographic characteristics of the west Mexican cotton rat (Sigmodon mascotensis). Mammalia, 74, 379-393.

http://dx.doi.org/10.1515/MAMM.2010.052

SEMARNAT (2010) NORMA Oficial Mexicana NOM-059-SEMARNAT-2010, Protección ambientalEspecies nativas de México de flora y fauna silvestres-Categorías de riesgo y especificaciones para su inclusión, exclusión o cambio-Lista de especies en riesgo. Disponible en: https://biblioteca.semarnat.gob.mx/janium/Documentos/Ciga/agenda/DOFsr/DO2454.pd f (Consultado: 02 junio 2021).

SEMARNAT-Instituto Nacional de Lenguas Indígenas (INALI) (2012) Riqueza lingüística y biológica de México. Cuadernos de divulgación ambiental. Toluca de Lerdo, 24 pp.

Torres, A., Luna-Vega, I. (2006) Análisis de trazos para establecer áreas de conservación en la Faja Volcánica Transmexicana. Interciencia, 31, 849-855.

Vane-Wright, R. I., Humphries, C. J., Williams, P. H. (1991) What to protect? Systematics and the agony of choice. Biological Conservation, 55, 235-254. http://dx.doi.org/10.1016/0006-3207(91)90030

Velázquez, A. (2012) El contexto geográfico de los lagomorfos de México. Therya, 3, 223-238. http://dx.doi.org/10.12933/therya-12-81

Weksler, M., Reis Percequillo, A., Voss, R. S. (2006) Ten new genera of Oryzomine rodents (Cricetidae: Sigmodontinae). American Museum Novitates, 3537, 1-29.

Whittaker, R. J., Araújo, M. B., Jepson, P., Ladle, R. J., Watson, J. E. M., Willis, K. J. (2005) Conservation biogeography: Assessment and prospect. Diversity and Distributions, 11, 323. https://doi.org/10.1111/j.1366-9516.2005.00143.x

Yáñez-Ordoñez, O., Trujano, M., Llorente-Bousquets, J. (2008) Patrones de distribución de las especies de la tribu Meliponini (Hymenoptera: Apidae) en México. Interciencia, 33, 41-45. 